\title{
X-ray Luminosity and Absorption Column Fluctuations in the H2O Maser Galaxy NGC 4258 from Weeks to Years
}

\section{Citation}

Fruscione, Antonella, Lincoln J. Greenhill, Alexei V. Filippenko, James M. Moran, James R. Herrnstein, and Elizabeth Galle. 2005. "X\#Ray Luminosity and Absorption Column Fluctuations in the H 20 Maser Galaxy NGC 4258 from Weeks to Years." The Astrophysical Journal 624 (1) (May): 103-117. doi:10.1086/428658.

\section{Published Version}

$10.1086 / 428658$

\section{Permanent link}

http://nrs.harvard.edu/urn-3:HUL.InstRepos:32085453

\section{Terms of Use}

This article was downloaded from Harvard University's DASH repository, and is made available under the terms and conditions applicable to Other Posted Material, as set forth at http:// nrs.harvard.edu/urn-3:HUL.InstRepos:dash.current.terms-of-use\#LAA

\section{Share Your Story}

The Harvard community has made this article openly available.

Please share how this access benefits you. Submit a story.

Accessibility 
Accepted by The Astrophysical Journal

\title{
X-ray Luminosity and Absorption Column Fluctuations in the $\mathrm{H}_{2} \mathrm{O}$ Maser Galaxy NGC 4258 from Weeks to Years
}

\author{
Antonella Fruscione ${ }^{1}$, Lincoln J. Greenhill ${ }^{1,2}$, Alexei V. Filippenko ${ }^{3}$, James M. Moran ${ }^{1}$, \\ James R. Herrnstein ${ }^{4}$, Elizabeth Galle ${ }^{1}$
}

\begin{abstract}
We report monitoring of the $0.3-10 \mathrm{keV}$ spectrum of NGC 4258 with the the XMM-Newton Observatory at five epochs over 1.5 years. We also report reprocessing of an overlapping four-epoch series of archival Chandra observations (0.5$10 \mathrm{keV}$ ). By including earlier $A S C A$ and Beppo-SAX observations, we present a new, nine-year time-series of models fit to the X-ray spectrum of NGC 4258. We model the Chandra and XMM-Newton data self-consistently with partially absorbed, hard power-law, soft thermal plasma, and soft power-law components. Over the nine years, the photoelectric absorbing column $\left(\sim 10^{23} \mathrm{~cm}^{-2}\right)$ did not vary detectably, except for a $\sim 40 \%$ drop between two $A S C A$ epochs separated by 3 years (in 1993 and 1996) and a $\sim 60 \%$ rise between two XMM-Newton epochs separated by just 5 months (in 2001 and 2002). In contrast, factor of 2-3 changes are seen in absorbed flux on the timescale of years. These are uncorrelated with changes in absorbing column and indicative of central engine variability. The most rapid change in luminosity $(5-10 \mathrm{keV})$ that we detect (with XMM-Newton and Chandra) is $\sim 30 \%$ over 19 days. The warped disk, a known source of $\mathrm{H}_{2} \mathrm{O}$ maser emission in NGC 4258, is believed to cross the line of sight to the central engine. We propose that the variations in absorbing column arise from inhomogeneities sweeping across the line of sight in the rotating disk at the radius where the disk crosses the line of sight. We estimate that the inhomogeneities
\end{abstract}

\footnotetext{
${ }^{1}$ Harvard-Smithsonian Center for Astrophysics, 60 Garden Street, Cambridge, MA 02138; afruscione@cfa.harvard.edu; greenhill@cfa.harvard.edu; moran@cfa.harvard.edu; egalle@cfa.harvard.edu

${ }^{2}$ Visiting physicist, Kavli Institute for Particle Astrophysics and Cosmology, Stanford Linear Accelerator Center, M.S. 29, 2575 Sand Hill Rd, Menlo Park, CA 94025

${ }^{3}$ Department of Astronomy, University of California, Berkeley, CA 94720-3411; alex@astro.berkeley.edu

${ }^{4}$ Renaissance Technologies, Inc., 600 Rt. 25A, E. Setauket, NY 11733; jrh@rentec.com
} 
are $\sim 10^{15} \mathrm{~cm}$ in size at the crossing radius of $0.29 \mathrm{pc}$, slightly smaller than the expected scale height of the disk. This result thus provides strong evidence that the warped accretion disk is the absorber in this (and possibly other) active galactic nuclei (AGNs). This is the first direct confirmation that obscuration in type-2 AGN may, in some cases, arise in thin, warped accretion disks, rather than in geometrically thick tori. Some previous studies report detection of weak Fe $\mathrm{K} \alpha$ emission in NGC 4258. We do not detect this line emission in any of our XMM-Newton spectra with a $90 \%$ upper limit to the equivalent width of $\sim 49$ eV for one observation. Weak, time-variable Fe line absorption has also been reported for a previous Chandra study. We do not observe evidence of absorption lines in the XMM-Newton or reprocessed Chandra data. The absence of Fe line emission is consistent with the disk being optically thin to hard photons as well as subtending a small solid angle as seen from the central engine because of the known shallowness of the warp.

Subject headings: accretion, accretion disks — black hole physics - galaxies: active — galaxies: individual (NGC 4258) — galaxies: Seyfert — X-rays: galaxies

\section{Introduction}

Radio interferometric imaging studies of $\mathrm{H}_{2} \mathrm{O}$ maser emission have directly demonstrated that, in at least a few cases, type-2 active galactic nuclei (AGNs) contain thin, nearly edge-on, warped accretion disks, with large columns of molecular gas inside $\sim 1$ pc. Examples include NGC 4258 (Miyoshi et al. 1995), NGC 1068 (Greenhill \& Gwinn 1997), and the Circinus galaxy (Greenhill et al. 2003). In these systems, the maser emission is likely stimulated by irradiation and heating of relatively cold gas by hard X-rays (e.g., Neufeld \& Maloney 1995). Because the structure of the maser emission can be readily resolved in velocity and angle, it has been possible to use it to map dense $\left(n_{\mathrm{H}_{2}} \approx 10^{9 \pm 1} \mathrm{~cm}^{-3}\right)$, warm $(T \approx 300-1000 \mathrm{~K})$ accretion-disk gas (Elitzur 1992).

The $\mathrm{H}_{2} \mathrm{O}$ source in the low luminosity $\left(L / L_{\text {Eddington }} \approx 10^{-4}\right)$ AGN of NGC 4258 (M106) is remarkable because the disk is extremely well described by a relatively simple dynamical model (Miyoshi et al. 1995). The maser emission has been mapped from radii of $0.14 \mathrm{pc}$ to $0.28 \mathrm{pc}$ around a $3.9 \times 10^{7} \mathrm{M}_{\odot}$ central mass. Maser positions, line-of-sight velocities and accelerations, and proper motions are all measurable, and they fit a simple geometrically thin, slightly warped, Keplerian disk model to high accuracy (Herrnstein et al. 1999). The knowledge of these quantities presents strong constraints for hard X-ray studies aimed at understanding processes related to the central engine: emission mechanisms and surrounding 
disk structure.

The structure and energetics of type-2 AGNs, in fact, are still not well understood mainly because X-ray spectra are complex superpositions of components due to several processes (compact and extended emission, absorption, scattering, and reflection with different ionization states) and most astronomical wavebands currently cannot resolve structures $<1 \mathrm{pc}$ in size. Many AGN models postulate that all AGN are intrinsically the same and orientation plus obscuration effects regulate their classification (e.g., Antonucci \& Miller 1985; Urry \& Padovani 1995; Elvis 2000). However, the actual nature of the obscuring material is still very much under debate, and several locations and structures have been proposed. These include a geometrically thick structure, galactic dust (Malkan et al. 1998), a geometrically thin absorber confined to radii comparable to the broad-line regions (e.g., Madejski et al. 2000; Risaliti et al. 2002), or an outflow (Elvis et al. 2000).

In the case of NGC 4258 the explanation for the unusually low luminosity is also an unsettled question: is it the result of a low accretion rate or radiatively inefficient accretion? Neufeld \& Maloney (1995) suggested the former. Other authors (e.g., Lasota et al. 1996; Gammie, Narayan, \& Blandford 1999) have modeled the global spectral energy distribution of NGC 4258 with advection-dominated, radiatively inefficient accretion flows (ADAF). Yuan et al. (2002) have suggested an alternative model in which outflow from the ADAF powers the jet in NGC 4258, the emission from which dominates the continuum from radio to X-ray wavebands. In each case the hard X-ray continuum is expected to arise from a different location (a corona above the disk, hot plasma centered on the central engine, or the base of a jet).

While early soft $(<2.5 \mathrm{keV})$ X-ray observations detected emission from NGC 4258 (Fabbiano, Kim, \& Trinchieri 1992; Pietsch et al. 1994), only ASCA X-ray observations in the hard band (2-10 keV) allowed Makishima et al. (1994) to model for the first time the spectrum of the central X-ray source of NGC 4258 as an absorbed power law, weak Fe $\mathrm{K} \alpha$ line, and optically thin soft thermal component. Reynolds, Nowak, \& Maloney (2000) report on later $A S C A$ observations and temporal variability on time scales of years in the absorbing column (30\%) and in the 5-10 keV flux (a factor of two). Observations with the Beppo-SAX PDS detected an extension of the power-law component to $70 \mathrm{keV}$ with no significant reflection component: NGC 4258 is one of the only five Seyfert 2 galaxies, among a total of 31, for which model fits to the Beppo-SAX data are not improved by the addition of a reflection component (Risaliti 2002); Beppo-SAX MECS observations detected a factor of two variations in count rate $(3-10 \mathrm{keV})$ on time scales $<100 \mathrm{ks}$ (Fiore et al. 2001), though Reynolds, Nowak, \& Maloney (2000) did not report variability over $200 \mathrm{ks}$ for a later epoch.

Observations of NGC 4258 with Chandra and XMM-Newton have been the first to 
separate the central compact emission component from the bulk of the extended soft emission that originates in well-known nonstellar spiral arms on kpc scales (e.g., Wilson et al. 2001). The first Chandra and XMM-Newton spectra are in agreement with ASCA and Beppo-SAX spectra, except that Fe K $\alpha$ emission is not detected, which has raised doubts about the original detections. Nonetheless, the line emission is presumed to be closely associated with the central engine, and non-detection may be indicative of variability in line equivalent width of a factor of two on time scales of 1-2 years. Time variability and narrow line-width would argue in favor of an association between the line emission and accretion-disk material (Reynolds, Nowak, \& Maloney 2000) at radii between $\sim 3 \times 10^{-4}$ and $\sim 1$ pc, either by reflection of light from a variable central source or by variation in disk structure (Pietsch \& Read 2002). Variability on shorter time scales ( $10 \mathrm{ks}$ ) has been claimed by Young \& Wilson (2004) in the possible detection of weak absorption features at 6.4 and $6.9 \mathrm{keV}$, which presumably would be caused by disk or outflow material at radii $\ll 1 \mathrm{pc}$.

We report in this paper the monitoring of NGC 4258 at five epochs with XMM-Newton (including the epoch reported by Pietsch \& Read 2002) and four epochs with Chandra (Young \& Wilson 2004) that are publically available. In total, the XMM-Newton and Chandra monitoring covers 2.2 years, 2000.2 to 2002.4. The primary goal of the program was characterization of time variability in the absorbing column that might be related to substructure of the accretion disk traced by maser emission. We present a time series of spectral models for NGC 4258, where we have minimized sources of systematic uncertainty by reprocessing and reanalyzing all existing Chandra and XMM-Newton datasets using a consistent set of models and techniques, as well as the most recent calibration databases. We describe the XMM-Newton and Chandra datasets, their calibration, and the spectral fitting in Section 2. We discuss our findings in Section 3. UT dates are used through this paper.

\section{Observations and Analysis}

\subsection{XMM-Newton Observations}

NGC 4258 was observed four times from 2001 May to 2002 May as part of an XMMNewton GO program. Table 1 lists the observation log for the EPIC instrument including the useful exposure times before and after filtering for high background times following the procedure outlined below. For completeness and consistency in analysis, we have included the first XMM-Newton observation of NGC 4258, obtained in 2000 December (Pietsch \& Read 2002).

All EPIC PN and MOS observations were taken in Prime Full Window mode with a 
Medium Filter. The entire data analysis was accomplished with a combination of tasks in the XMM-Newton and Chandra data analysis software systems (Science Analysis Software - SAS - versions 20030110_1802/20040318_1831 and Chandra Interactive Analysis of Observations - CIAO - versions 3.0.1/3.0.2). We generated calibrated EPIC event files using the epproc and emproc routines and filtered the final event list to include only "good" events, with patterns 0-4 (singles and doubles) for the PN camera and 0-12 for the MOS cameras. We also rejected bad times due to high background flares (Figure 1) through a comparison of the source and background light curves for each observation. Ratios of source and background count rates were calculated for each $100 \mathrm{~s}$ time-bin, and we eliminated all time intervals with source-to-background count ratios lower than 5. Useful integration times were reduced as shown in Table 1. The observation most affected by overall high background levels as well as strong flares was on 2001 Dec. 17 (Figure 1). We compared the expected and observed distributions of event grades calculated with the SAS routine epatplot to quantify the effects of event pile-up, which were not detectable. ${ }^{1}$

Spectra were extracted using the SAS routine especget excluding events near the edge of the CCDs and near bad pixels which may have incorrect energies. We chose a circular source extraction region of 300 pixel radius ( or $15^{\prime \prime}$ ) which covers about $80 \%$ of the PSF and a background extraction region of 1200 pixel radius $\left(60^{\prime \prime}\right)$ lying $\sim 6^{\prime}$ from the source on the same chip as the source region. We binned the spectral pulse-invariant (PI) channels by a factor of 10 in all spectra to obtain uniform binning while increasing the statistics in each individual bin (Figures 2-6).

The most recent response matrices (version of 2003-01-29) for each instrument observing mode and observation date were obtained from the XMM-Newton calibration archive and used in the spectral fitting. The same source and background extraction regions were used for deriving the light curves (Figure 1) for the PN and MOS cameras. All light curves were extracted over the entire energy range (nominally $0.2-15 \mathrm{keV}$ ) taking into account an average dead-time correction calculated from the livetime and ontime keywords recorded in the data. Average count rates for all observations are listed in Table 1.

We note in passing that we also checked the XMM-Newton RGS spectra which, however, provided no additional constraints to the spectral modeling because of the extremely poor signal-to-noise ratio in the covered waveband $(<2.5 \mathrm{keV})$.

\footnotetext{
${ }^{1}$ When two (or more) photons are detected in a single pixel within one read-out period, they are perceived to be a single, "piled-up" event, and the count rate is depressed. The resulting distribution of grades and the energy spectrum are distorted since the apparent energy is approximately the sum of the photon energies.
} 


\subsection{Chandra Observations}

The Chandra ACIS-S camera observed NGC 4258 four times from 2000 March to 2001 May (Table 2) with useful exposure times ranging from $\sim 1.6$ to $\sim 20 \mathrm{ks}$, as described by Pietsch \& Read (2002) and Young \& Wilson (2004). The 2000 April 17 (13 ks) and 2001 May 29 (20 ks) observations were optimized to study the faint "anomalous arms" of NGC 4258. As a result, both suffer from significant photon pile-up in the nuclear region, of order $20-30 \%$. Detailed modeling was needed to account for this effect when fitting spectra (see $\S 2.3 .1)$.

We re-analyzed all Chandra data to ensure that the most recent calibrations were used and that the analysis techniques were consistent with those used for the XMM-Newton data. We reprocessed the data using the Chandra calibration database (CALDB) versions 2.23 and 2.26 and CIAO software version 3.0.1 and 3.0.2. In the data preparation phase we checked for background flares (none present in any of the data sets) and defined extraction regions: a $2^{\prime \prime}$ radius source region on the nucleus and a $10^{\prime \prime}$ radius background region about $2^{\prime}$ from the nucleus. Responses were derived from the latest calibration data available in CALDB 2.26 and included the effects due to a reduction of the low-energy quantum efficiency of the ACIS instrument ${ }^{2}$. As in the XMM-Newton analysis, extracted spectra were binned by a factor of 10 in spectral PI channels (Figures 7, 8, 11, 12). In some cases we verified the results by also binning to $\geq 15$ counts per bin for comparison.

\subsection{Spectral Analysis}

We used version 3.0.2 of Sherpa (Freeman, Doe, \& Siemiginowska 2001) to fit models to our spectra iteratively, widening the energy interval being fit and adding model components as required to improve the significance of the spectral fit (Figures 2-8, 11, 12) .

For the XMM-Newton data we started by fitting the 2-10 keV energy range with a simple power-law model, absorbed by both intrinsic absorption and Galactic absorption $\left(N_{H}\right)$, the latter fixed at the value $1.19 \times 10^{20} \mathrm{~cm}^{-2}$ (Murphy et al. 1996). We verified that the background is negligible for all observations, so we neither subtracted it nor modeled it during the spectral fitting. We then extended the energy range to $0.3 \mathrm{keV}$, adding a thermal plasma component (the "mekal" model: Mewe, Gronenschild, \& vad den Oord 1985; Mewe, Lemen, \& van den Oord 1986; Kaastra 1992; Liedahl, Osterheld, \& Goldstein 1995) with elemental abundances as a fixed fraction of the cosmic values (Anders \& Grevess 1989), and

\footnotetext{
${ }^{2}$ More details can be found at http://cxc.harvard.edu/cal/Acis/Cal_prods/qeDeg/.
} 
a soft power-law component, both absorbed by the Galactic $N_{H}$. The best-fit values for all the free parameters in the models are listed in Table 2, together with model-derived fluxes and luminosities in various energy ranges, assuming a distance of 7.2 Mpc (Herrnstein et al. 1999) so $1^{\prime \prime}=35 \mathrm{pc}$.

In order to assess the quality of the fit we used the $\chi^{2}$ statistic proposed by Kearns, Primini, \& Alexander (1995) and implemented in Sherpa (so-called "chi primini" statistic) which is appropriate for non-background-subtracted data. This statistic can be used even when the number of counts in each bin is small $(<5)$ and it is not biased (see, e.g., Freeman 2001).

Spectral fits obtained with a bremsstrahlung component instead of a soft power-law component work equally well at soft energies and neither has a significant influence on the best-fit parameters for the direct AGN component (both in term of slope and absorption). These soft components may reflect some integrated Galactic contribution, such as from the $\mathrm{X}$-ray binary population. The thermal plasma component is needed to account for the strong iron $\mathrm{L}$ complex detected at $\sim 1 \mathrm{keV}$.

For the Chandra observations the background was also negligible, so we neither subtracted it nor modeled it. However, in any case, we note that background subtraction is not recommended (Davis 2001) for data to which pile-up models will be applied. We again started the analysis by fitting a simple absorbed power-law model in the $2-10 \mathrm{keV}$ energy range. We then added two model components to extend the fit to $0.5 \mathrm{keV}$. Finally, for the two longest Chandra observations, we added a pile-up model to the fit (see below). The "chi primini" statistic was used for the two shortest Chandra observations, while the "chi dvar" statistic (a $\chi^{2}$ statistic with variance computed from the observed counts data) was preferred for the two longest piled-up Chandra observations for computer efficiency reasons.

Inspection of the residuals to the best fits to XMM-Newton and Chandra data did not reveal the presence of statistically significant emission or absorption lines in any of the spectra. To calculate upper limits to $\mathrm{Fe} \mathrm{K} \alpha$ emission line equivalent widths, we added a Gaussian component to the $2-10 \mathrm{keV}$ power-law model (Table 2), fixing the line energy $\left(E_{\text {line }}\right)$ at $6.4 \mathrm{keV}$ and obtaining upper limits to the equivalent widths $\left(W_{\text {line }}\right)$ using the estimated upper bound of the uncertainty in line normalization (90\% confidence). In one case (the 2001 June 17 XMM-Newton observation) a line can be fit, if $E_{\text {line }}$ is allowed to vary (Table 2); however, the fit is only improved at $<2 \sigma$ level by the addition of the Gaussian model component (reduced $\chi^{2}=1.05$ for 159 degrees of freedom without a line, reduced $\chi^{2}=1.02$ for 156 degrees of freedom with a line), which we therefore do not classify as a detection. 


\subsubsection{Pile-up Modeling of Chandra Data}

As noted earlier, two of the Chandra observations (on 2000 April 17 and 2001 May 28) were strongly affected by photon pile-up. We accounted for this at the spectral fitting level by including the model by Davis (2001), as implemented in Sherpa, in our overall spectral fit. Following the instructions for using the model for Chandra data we

(i) re-included all events that the automated pipeline classifies as "afterglow" events before performing the spectral extraction,

(ii) verified the constancy of the source during the observation,

(iii) extracted the spectra from a $2^{\prime \prime}$ radius source region around the nucleus, and

(iv) did not subtract the background in the fitting process.

The Davis (2001) pile-up model is described by various parameters, two of which are generally the most crucial: $\alpha$ (which represents the probability of getting a "good" event grade - essentially a non-background event — when two photons pile together), and $f$ (the fraction of flux falling into the pile-up region). To investigate systematic errors that may result from the details of how pile-up is modeled, we processed each pile-up epoch in three ways: with $\alpha$ and $f$ free to vary, $\alpha$ free and $f$ fixed, and $\alpha$ and $f$ both fixed. The results of the fits are reported in Table 3. For both epochs the three pile-up corrected models are statistically consistent within the $90 \%$ errors and the global pile-up fraction (i.e. the estimated percentage of observed counts containing two or more photons piled together) is not significantly affected by the choice of $\alpha$ and $f$ parameters. However, we note a systematic bias in parameters when either $\alpha$ or $f$ are held fixed (e.g., we obtain a steeper photon index, $\left.\Gamma_{p l}\right)$. We also note better agreement with the non-piled-up Chandra epochs, and lower reduced $\chi^{2}$, when $\alpha$ and $f$ are allowed to vary during the fit. For this reason we adopt "unfrozen" models and from a comparison of parameters listed in Table 2 suggest that the systematic uncertainties in $L_{2-10}, \Gamma_{p l}$, and $N_{H}$ are on the order of $20 \%, 20 \%$, and $10 \%$. (Hard absorbed flux is not significantly affected.) Differences between our best-fit parameters and those of Young \& Wilson (2004) are probably in part related to the treatment of pile-up ${ }^{3}$.

\footnotetext{
${ }^{3}$ See also Nowak 2003 at http://space.mit.edu/CXC/analysis/PILECOMP/ for a detailed discussion of modeling piled-up spectra with high-energy tails.
} 


\subsection{Comparison of Results Obtained for Different Missions}

In Table 2 we report a cumulative record of spectral parameters from the literature and obtained by us in processing new and archival Chandra and XMM-Newton datasets. Care in comparison of results obtained with different instruments is warranted because of different angular and energy resolutions, energy coverage, and cross-calibration effects (see, e.g., Marshall et al. 2001 on Chandra-XMM-Newton cross-calibration). For example, analysis of Beppo-SAX data (extraction radius $3^{\prime}$ in the $2-10 \mathrm{keV}$ band) has delivered the steepest $\Gamma_{p l}$, and Beppo-SAX was effectively the only mission sensitive to emission above $\sim 10 \mathrm{keV}$. Analyses of Chandra data consistently provide the shallow estimates of $\Gamma_{p l}$ and low estimates of $N_{H}$ (for epochs free of pile-up and epochs where pile-up parameters are free to vary; see, e.g., Figure 13). This may in part be a consequence of angular resolution - in other words, a small extraction radius $\left(2^{\prime \prime}\right.$ or $\left.\sim 70 \mathrm{pc}\right)$ better isolates the central engine from surrounding soft nuclear emission. (XMM-Newton supports the next smallest extraction radius, $15^{\prime \prime}$ or $\sim 520$ pc.) On the other hand, hard and soft components can be disentangled in analysis of somewhat lower angular resolution data (e.g., XMM-Newton) if the source model is accurate. In that case, the limited effective collecting area of Chandra above $\sim 6 \mathrm{keV}$ may be a more significant factor, as it reduces constraints on the hard portion of the spectrum and increases correlations between fit parameters (such as $N_{H}$ and $\Gamma_{p l}$ ). In contrast, XMM-Newton count rates are higher, provide better constraint on the hard portion of the spectrum, and probably provide more robust parameter estimates (for this galaxy).

\section{Discussion}

We have added four new epochs to the monitoring record of hard X-ray emission from NGC 4258 which now extends to 9 years. The five XMM-Newton observations by themselves provide a much needed time-series of $0.3-10 \mathrm{keV}$ spectra with high count rate and spectral resolutions that are unaffected by serious instrumental systematics, such as pile-up. The time separations between XMM-Newton epochs span 0.12 to 1.5 years. Taken together, the time series of Chandra and XMM-Newton epochs spans 2.2 years with a minimum separation of 0.05 years ( 3 weeks).

The source models fit to the XMM-Newton data are on average consistent with the range of models fit to data from other satellites. For the five XMM-Newton epochs, we obtain an absorbing column $N_{H}=(8.6-13.2) \times 10^{22} \mathrm{~cm}^{-2}$, absorption-corrected luminosity $L_{X}=(5.4-9.1) \times 10^{40} \operatorname{ergs~s}^{-1}(2-10 \mathrm{keV})$ or $L_{X}=(2.9-4.4) \times 10^{40} \mathrm{ergs} \mathrm{s}^{-1}(5-10 \mathrm{keV})$, photon index $\Gamma_{p l}=1.5-1.7$, and thermal plasma temperature $k T=0.5-0.6 \mathrm{keV}$ (Table 2). The characteristics of the soft emission component do not in general display substantial 
range, and the XMM-Newton results are statistically consistent with estimates reported elsewhere.

\subsection{Continuum Variability}

Count rates and estimates of absorbed flux $\left(f_{X}\right)$ obtained just with XMM-Newton (2$10 \mathrm{keV}$ and $5-10 \mathrm{keV}$ ) demonstrate intensity variations of $10-30 \%$ on time scales as short as six months (Figures 1, 14; Table 2). However, comparing XMM-Newton and Chandra data that are unaffected by pile-up, we observe a $\sim 30 \%$ decrease in hard (5-10 keV) absorbed flux between epochs separated by 19 days (2001 May 29 and 2001 Jun 17). Count rates estimated by Young \& Wilson (2004) also appear to indicate variation between two epochs separated by $\sim 1$ month (2000 March 8 and 2000 April 17). However, interpretation of this comparison requires caution because of systematic uncertainty associated with pile-up modeling applied to the later epoch (see §2.3.1).

Evidence of more rapid variability has been reported, but the contributions of systematics can be difficult to assess, such as pile-up (in Chandra data), background variability, or the presence of other variable sources in the extraction region. For example, Fiore et al. (2001) report Beppo-SAX light-curve variations of 10-20\% on time scales of $\sim 1$ hour and $100 \%$ on time scales on the order of half a day, but without background subtraction and for an extraction region of 3 ' (which includes, for example, the soft companion discovered by Chandra $\sim 3^{\prime \prime}$ to the southwest of the nucleus; Pietsch \& Read 2002). In contrast, on time scales of $\sim 3$ hours, we observe with XMM-Newton no variability appearing in background-subtracted light curves for both the PN and MOS instruments (Figure 1). Similarly, Reynolds, Nowak, \& Maloney (2000) observe no variability with $A S C A$ on scales up to $\sim 2$ days.

We also detect variability in unabsorbed luminosity $\left(L_{X}\right)$ with XMM-Newton (Figure 14). In the $\sim 1$ year prior to the last epoch, $L_{X}(2-10 \mathrm{keV})$ dropped $\sim 20 \%$ to $\sim 5.4 \times 10^{40} \mathrm{erg} \mathrm{s}^{-1}$, possibly as part of a long-term decline by approximately a factor of two from the peak detected by Chandra on 2000 March 8. This large variation is comparable in magnitude to what has been seen for ASCA, Beppo-SAX, and early Chandra epochs (Figure 14), when $L_{X}(2-10 \mathrm{keV}$ ) varied by factors of $\sim 2$ (see also Reynolds, Nowak, \& Maloney 2000). We exclude the possibility that cross-correlation in the modeling causes these variations, referring to Figure 14 and the absence of any correlation between estimates of $L_{X}$ and $N_{H}$. (We recall that $N_{H}$ and $\Gamma$ appear to be somewhat correlated among models for Chandra data (see $\$ 2.3 .2$ ), but emphasize that over a wide range, variations in $L_{X}$ do not appear to be correlated with changes in either $N_{H}$ or $\Gamma$.) 
Although there is no one-to-one correspondence, we note that two of the lowest estimates of $L_{X}$ are associated with the two largest estimates of $N_{H}$. Within the $\sim 5$ months prior to the last XMM-Newton epoch, $N_{H}$ increased $\sim 50 \%$, returning to a high level $[(1.3 \pm$ $\left.0.1) \times 10^{23} \mathrm{~cm}^{-2}\right]$ not reported since the first $A S C A$ observations in 1993. In both cases, $L_{X}(5-10 \mathrm{keV})$ is $<4 \times 10^{40} \mathrm{ergs} \mathrm{s}^{-1}$. We speculate that reductions in $L_{X}$ might affect the ionization state of the absorber, resulting in increased columns in some cases, though we acknowledge that NGC 4258 is a weak source, and it is difficult to establish a connection. If true, then a change in ionization would suggest that the absorber is not entirely neutral.

\subsection{Absorbing Gas}

NGC 4258 is an unusual target to model because the central mass, geometry, and dynamics of surrounding dense material are accurately known from modeling of radio interferometry data of the maser emission. The best-fit warped disk model, which is derived from observed maser positions and Doppler velocities, is highly inclined. In particular, the warp of the disk brings it across the line of sight to the dynamical center at a radius of $0.29 \mathrm{pc}$, where its angle to the line of sight is about $16^{\circ}$, just beyond the outer limit of the known maser emission (?), and thus placing high-density gas $\left(\sim 10^{9 \pm 1} \mathrm{~cm}^{-3}\right.$; Elitzur 1992) between us and the central engine.

To interpret the time variability in the absorption column density, we assume that inhomogeneities in the disk move across our line of sight as the disk rotates and that these are responsible for observed changes in absorption. The discussion that follows depends rather critically on the view that the maser emission is a good tracer of the disk - i.e., that its emission defines the mid-plane of the disk, and that the disk changes from molecular to atomic beyond a radius of about 0.28 pc, as described by Neufeld \& Maloney (1995). For these assumptions, the mass accretion rate is about $10^{-4} \alpha^{-1}$ solar masses per year.

We estimate the physical size of the inhomogeneities in the disk from the variability time scale of the absorption column density. The rotation speed of the disk at a radius of $0.29 \mathrm{pc}$ is about $v_{\text {rot }}=760 \mathrm{~km} \mathrm{~s}^{-1}$ for a central mass of $3.9 \times 10^{7} \mathrm{M}_{\odot}$. Hence the length scale $\left(r_{c}\right)$ associated with the observed time-variability scale $(\tau)$ of 5 months is $r_{c}=v_{\text {rot }} \tau$, or about $1.0 \times 10^{15} \mathrm{~cm}$. For the standard thin accretion disk (e.g., Frank, King, \& Raine 1992), the scale height (h) obeys the relation $h=c_{s} r^{3 / 2}(G M)^{-1 / 2}$. If the gas is atomic at the line-of-sight crossing radius, then the temperature is expected to be about $8000 \mathrm{~K}$ and $c_{s}=7 \mathrm{~km} \mathrm{~s}^{-1}$, according to the model of Neufeld \& Maloney (1995). Hence, $\mathrm{h}=8 \times 10^{15} \mathrm{~cm}$ and $r_{c} \approx h / 8$. If the gas at the crossing radius were molecular, and at the preferred maser temperature of about $800 \mathrm{~K}$, the sound speed would be about $2.3 \mathrm{~km} \mathrm{~s}^{-1}$, and the scale 
height would then be about $1.1 \times 10^{15} \mathrm{~cm}$, close to $r_{c}$. However, if the gas were atomic, then the mass accretion rate, which scales as $c_{s}^{2}$, would have to be about $10^{-5} \alpha^{-1} \mathrm{M}_{\odot} \mathrm{yr}^{-1}$. This would move the atomic transition radius into the maser region, and also make the density at the inner radius of maser emission unacceptably low. We note that the time scale of bright maser flares is about 2 months (Herrnstein et al. 2005). At the radius of the flare maser, $0.17 \mathrm{pc}$, the rotation speed is about $970 \mathrm{~km} \mathrm{~s}^{-1}$, the scale for density fluctuations is $5 \times 10^{14}$ $\mathrm{cm}$, and the scale height is $1.2 \times 10^{14} \mathrm{~cm}$. Hence, here also the turbulence scale length is somewhat smaller than the local scale height.

Advective accretion models have been proposed for NGC 4258 (e.g., Gammie, Narayan, \& Blandford 1999, and references therein) which require much larger accretion rates and much thicker disks, and for which the masers are relegated to a thin surface skin. The difficulty with these models is the much more massive disk required. The gross model geometry of the disk would not change, still crossing the line of sight to the center at the same radius. However, the column density would be much greater at the crossing radius than determined by the X-ray observations.

Three other reservoirs for absorbing gas are also conceivable: an edge-on molecular torus at much larger radii than the "maser disk" (as is often assumed in the AGN unification paradigm), an outflow (e.g., Elvis 2000), and an extension of the disk to much smaller radii. We argue against the presence of a massive torus because the $\mathrm{CO}$ distribution in NGC 4258 is not centrally peaked (Plante et al. 1991, and references therein). We argue against outflow because NGC 4258 is a low-luminosity AGN and the possibility of it driving a massive outflow seems remote. In addition, radio and optical imaging show a well-collimated flow (Herrnstein et al. 1997; Cecil et al. 2000) that lies well away from the line of sight to the central engine. The possibility of absorption arising in a disk-like structure at small radii ( $\ll 0.14 \mathrm{pc}$ ) exists, but is unlikely given the three-dimensional geometry of the maser disk. We note that the disk inclination is $\sim 82^{\circ}$ at a radius of 0.14 pc. For an $m=1$ warping mode, the inclination is more face-on at smaller radii (down to $\sim 74^{\circ}$ ). Hence, disk material at small radii cannot lie along the line of sight to the central engine unless the disk is significantly thickened $(h / r>0.3)$. If the absorbing material is neutral and cold, this raises the question of what process provides vertical support. The problem of support is mitigated if the disk is very hot, but the X-ray spectrum of NGC 4258 does not show signs of very hot gas. 


\section{3. $\quad \mathbf{F e} \mathbf{K} \alpha$ Emission Line}

We do not detect Fe $\mathrm{K} \alpha$ line emission in any of the XMM-Newton epochs or in any of the Chandra epochs. The XMM-Newton observation on 2001 May 6 puts a stringent $90 \%$ upper limit to the equivalent width of $<49 \mathrm{eV}$. In light of the last detection of the line with $A S C A$ at epoch 1999 May 15 (Table 2), Pietsch \& Read (2002) and Reynolds \& Nowak (2003) concluded the line is variable, fading on a time scale of $<10$ months. The apparent disappearance, which now extends through May 2002, is difficult to explain, assuming the earlier detections with $A S C A$ (at multiple epochs) were robust.

The accretion disk is the most prominent reservoir of cold material known in close proximity to the central engine. Assuming that the line originates in the disk, its weakness is consistent with the inferred relatively low column density of the disk $\left(\sim 10^{23} \mathrm{~cm}^{-2}\right)$, which enables transmission of incident hard X-rays (i.e., $6.4 \mathrm{keV}$ ) virtually unattenuated. As a result, reflection should be negligible (see also Fiore et al. 2001), and Ghisellini, Haardt, \& Matt (1994) have argued that at such low column densities, equivalent widths for line fluorescence should be $\ll 100 \mathrm{eV}$.

The weakness of the line is also consistent with the disk subtending a small solid angle $(\Omega / 2 \pi \ll 1)$ as seen from the source of hard X-ray continuum near the disk center. This would be the case if NGC 4258 were to host an advection-dominated accretion flow (ADAF). Herrnstein et al. (1997) measured the angular structure of radio continuum emission in the vicinity of the disk center, and showed that any ADAF must be compact, $<100$ Schwarzschild radii. No cold material would extend toward the center, and as a result the disk solid angle would be small. Although the presence of an ADAF could explain the weakness of Fe line emission, our earlier argument that a large mass accretion rate and thick disk are inconsistent with the observed X-ray absorbing column remains valid. In addition, we note that Blandford \& Begelman (1999) suggest that ADAFs should be accompanied by massive winds (thus depleting the accretion flow), and Fiore et al. (2001) argue that such winds are inconsistent with the broadband spectral energy distribution of NGC 4258. In place of an ADAF as the source of hard X-ray continuum emission, a disk corona remains a possibility. Although in this case the disk could extend inward far enough that it would subtend a large solid angle $(\Omega / 2 \pi \approx 1)$, the relative (optical) thinness of the disk alone could explain the weakness of any $\mathrm{Fe} \mathrm{K} \alpha$ emission. It also remains possible that the line emission arises from scattering, fluorescence, or reflection by material that lies away from the disk, but apart from the well-collimated radio synchrotron jet (Herrnstein et al. 1997), none is known.

Past time variability of the line is more difficult to explain: light travel time arguments applied to the fading suggest that the line-emitting region is $<0.5 \mathrm{pc}$ in size, corresponding to the approximate outer diameter of the maser disk. The changes in the line may be the 
result of changes in the disk across its surface area or changes in the central illumination. However, if both X-ray and radio (maser) line emission are the result of irradiation of the disk surface, then it is notable that the brightness and velocity extent of $\mathrm{H}_{2} \mathrm{O}$ maser emission have not changed substantially since monitoring began in 1992 (e.g., Nakai, Inoue, \& Miyoshi 1993; Bragg et al. 2000; Argon et al. 2005). This would suggest that the disk (at 0.14$0.28 \mathrm{pc}$ radii) and its source of illumination have remained relatively unchanged over the period that the Fe line has faded. However, considering the fact that both maser emission and Fe line emission may be influenced by other effects (e.g., propagation along the disk, gas heating and cooling rates), coordinated X-ray and radio monitoring over time scales of $\lesssim 1$ day to $\sim 6$ months is needed to more firmly detect the line and to test proposed emission and variability mechanisms.

\subsection{Iron Absorption Lines}

Time-variable Fe line absorption (at 6.4 and $6.9 \mathrm{keV}$ ) has been reported from a study of one (2000 April 28) of the Chandra observations by Young \& Wilson (2004). In our analysis we find no evidence of absorption lines, neither in the XMM-Newton nor in the Chandra spectra. Because our findings differ from what was previously reported, we tested our results by retracing, to the best of our knowledge, these authors' data-analysis steps. We repeated the analysis using several binning schemes for the extracted spectra (including a minimum of 15 counts per bin as used by the previous authors), we inspected the data split in two equal time intervals, and we applied pile-up models with various choices of parameters. Figures 8 to 10 illustrate our results: no significant absorption feature can be inferred from our data and best-fit models. We can only speculate about possible origins for the discrepancy: differences in calibration, or slight differences in the data preparation, treatment of the background, or the modeling of pile-up. We note that the 2000 April 28 epoch is the one most affected by photon pile-up at a level of $\sim 30 \%$ (see Table 3 and §2.3.1).

\section{Summary and Conclusions}

In this paper we have analyzed X-ray $(0.3-10 \mathrm{keV})$ observations of NGC 4258 obtained with the XMM-Newton and Chandra observatories over $\sim 2$ years. Including earlier observations by $A S C A$ and Beppo-SAX, we present for the first time a new nine-year time-series of models fit to the X-ray data from NGC 4258.

Spectral and time variability analyses lead to the following main results: 
(1) The XMM-Newton and Chandra spectra of NGC 4258 data are well fit by a model consisting of several components: a partially absorbed, hard $(>2 \mathrm{keV})$ power law, a soft thermal plasma, and a soft power law. The soft emission, some of which arises $\lesssim 70$ pc from the central engine, does not vary appreciably from observation to observation.

(2) From XMM-Newton data, which are free of systematics such as pile-up, we observe long-term time variability in the source count rate and absorbed flux over time scales of $\sim 6$ months. No evidence for variability, however, is detected within our individual $\sim 3$ hour integrations.

(3) From XMM-Newton data we detect a $\sim 60 \%$ increase in $N_{H}$ over $\sim 5$ months, returning to a high level not reported since $A S C A$ observations in $1993, N_{H} \approx 1.3 \times 10^{23} \mathrm{~cm}^{-2}$.

(4) Changes in $N_{H}$ and $f_{X}$ are not correlated, which indicates intrinsic variability of the central engine that is in one case $30 \%$ over 19 days $(5-10 \mathrm{keV})$. We note that two of the largest estimates of unabsorbed luminosity are associated with the lowest estimates of $N_{H}$, and we speculate that reductions in $L_{X}$ might affect the ionization state of the absorber.

(5) The geometry and orientation of the accretion disk in NGC 4258 is well known from interferometric mapping of maser emission that arises in the accretion disk. We infer that the disk is somewhat inhomogeneous, based on observed time variability in $N_{H}$ and an assumption that the absorbing gas lies in the disk. Using the rotation curve of the disk, we estimate that the absorbing gas may lie at the same radius as where the disk is (independently) believed to cross our line of sight to the central engine (because the disk is warped) and that the inhomogeneities are $\sim 10^{15} \mathrm{~cm}$ in size.

(6) We do not detect Fe K $\alpha$ line emission in any of the XMM-Newton or Chandra epochs, thus extending the "disappearance" of the line from the last $A S C A$ detection in 1999 May to 2002 May. The inferred line emission region is comparable in size to the maser disk. If the line arises from the disk (e.g., by fluorescence), then it is difficult to understand the variability because the maser emission has not changed substantially.

(7) We do not observe evidence for absorption lines in any XMM-Newton or Chandra spectra.

Our inference that the accretion disk is inhomogeneous at radii of $\sim 0.3 \mathrm{pc}$ raises the question of what mechanism is responsible. Assessment of the Toomre Q-parameter at this radius suggests that self-gravity is unimportant except at the highest possible densities supportive of maser action (cf. Maoz 1995). Magnetic fields may play a role in confining inhomogeneities, but the field strength and configuration are not known (Herrnstein et al. 1998; Modjaz et al. 2005). While the confinement mechanism is not determined, we note 
that detection of inhomogeneities in the disk empirically supports techniques to estimate a distance to NGC 4258 from maser proper motions and centripetal accelerations, because these rely on the apparent maser dynamics tracking the rotation of disk material (Herrnstein et al. 1999, and references therein).

We thank Tom Aldcroft, Jeremy Drake, Martin Elvis, Greg Madejski, Guido Risaliti, and Aneta Siemiginowska for helpful discussions and insightful comments. AF and LJG are grateful to Martin Elvis for fostering their collaboration. This work has been supported by NASA GO grants NAG5-10194 and NAG5-11689, and by NASA science grant NAS8-39073 to the Chandra X-Ray Center.

\section{REFERENCES}

Anders, E., \& Grevesse N. 1989, Geochimica et Cosmochimica Acta 53, 197

Antonucci, R. R. J., \& Miller, J. S. 1985, ApJ, 297, 621

Argon, A. L., Greenhill, L. J., Humphreys, E. M., Reid, M. J., \& Moran, J. M. 2005, ApJ, in prep.

Blandford, R. D., \& Begelman, M. C. 1999, MNRAS, 303, L1

Bragg, A. E., Greenhill, L. J., Moran, J. M., \& Henkel, C. 2000, ApJ, 535, 73

Cecil, G., et al. 2000, ApJ, 536, 675

Davis, J. E. 2001, ApJ, 562, 575

Elitzur, M. 1992, Astronomical Masers (Dordrecht: Kluwer)

Elvis, M. 2000, ApJ, 545, 63

Fabbiano, G., Kim, D.-W., \& Trinchieri, G. 1992, ApJS, 80, 531

Fiore, F., et al. 2001, ApJ, 556, 150

Frank, J., King, A. R., \& Raine, D. J. 1992, "Accretion Power in Astrophysics," 2nd ed., (Cambridge: Cambridge University Press)

Freeman, P. E. 2001, "Issues in Model Fitting in the X-Ray Regime," GSFC X-Ray School, http://cxc.harvard.edu/sherpa/documents/presentations/freemanI.pdf 
Freeman, P. E., Doe, S., \& Siemiginowska, A. 2001, SPIE Proc., 4477, 76

Gammie, C. F., Narayan, R., \& Blandford, R. 1999, ApJ, 516, 177

Ghisellini, G., Haardt, F., \& Matt, G. 1994, MNRAS, 267, 743

Greenhill, L. J., \& Gwinn, C. R. 1997, Ap\&SS, 248, 261

Greenhill, L .J., et al. 2003, ApJ, 590, 162

Herrnstein, J. R., Moran, J. M., Greenhill, L. J., Blackman, E. G., \& Diamond, P. J. 1998, ApJ, 508, 243

Herrnstein, J. R., Moran, J. M., Greenhill, L. J., Diamond, P. J., Miyoshi, M., Nakai, N., \& Inoue, M. 1997, ApJ, 475, L17

Herrnstein, J. R., et al. 1999, Nature, 400, 539

Herrnstein, J. R., Moran, J. M., Greenhill, L. J., \& Trotter, A. S. 2005, in preparation

Kaastra, J. S. 1992, An X-Ray Spectral Code for Optically Thin Plasmas (Internal SRONLeiden Report, updated version 2.0)

Kearns, K., Primini, F., \& Alexander, D. 1995, in "Astronomical Data Analysis Software and Systems IV," ed. R. A. Shaw, et al. (San Francisco: ASP), 331

Lasota, J.-P., Abramowicz, M. A., Chen, X., Krolik, J., Narayan, R., \& Yi, I. 1996, ApJ, 462,142

Liedahl, D. A., Osterheld, A. L., \& Goldstein, W. H. 1995, ApJ, 438, L115

Maoz, E. 1995, ApJ, 455, L131

Madejski, G. M., Zycki, P. T., Done, C., Valinia, A., Blanco, P., Rothschild, R., \& Turek, B. 2000, ApJ, 535, L87

Makishima, K., et al. 1994, PASJ, 45, L77

Malkan, M. A., Gorjian, V., \& Tam, R. 1998, ApJS, 117, 25

Marshall, H. L., et al. 2002, "Cross-Calibrating Chandra with XMM-Newton," 1st Chandra Calibration Workshop, http://cxc.harvard.edu/ccw/proceedings/02_proc/presentations/h_marshall/cross_cal/

Mewe, R., Gronenschild, E. H. B. M., \& van den Oord, G.H.J. 1985, A\&AS, 62, 197 
Mewe, R., Lemen, J. R., \& van den Oord, G. H. J. 1986, A\&AS, 65, 511

Miyoshi, M., Moran, J., Herrnstein, J., Greenhill, L., Nakai, N., Diamond, P., \& Inoue, M. 1995, Nature, 373, 127

Modjaz, M., Moran, J. M., Greenhill, L. J., Kondratko, P. T. 2005, ApJ, submitted.

Moran, J., Greenhill, L., Herrnstein, J., Diamond, P., Miyoshi, M., Nakai, N., \& Inoue, M. 1995, PNAS, 92, 11427

Murphy, E. M., Lockman, F. J., Laor, A., \& Elvis, M. 1996, ApJS, 105, 369

Nakai, N., Inoue, M., \& Miyoshi, M. 1993, Nature, 361, 45

Neufeld, D. A., \& Maloney, P. R. 1995, ApJ, 447, L17

Neufeld, D. A., Maloney, P. R., \& Conger, S. 1994, ApJ, 436, L127

Pietsch, W., \& Read, A. M. 2002, A\&A, 384, 793

Pietsch, W., Vogler, A., Kahabka, P., Jain, A., \& Klein, U. 1994, A\&A, 284, 386

Plante, R. L., Lo, K. Y., Roy, J., Martin, P., \& Noreau, L. 1991, ApJ, 381, 110

Reynolds, C. S., \& Nowak, M. A. 2003, Phys. Reports, 377, 389

Reynolds, C. S., Nowak, M. A., \& Maloney, P. R. 2000, ApJ, 540, 143

Risaliti, G., Elvis, M., \& Nicastro, F. 2002, ApJ, 571, 234

Urry, C. M., \& Padovani, P. 1995, PASP, 107, 803

Wilson, A. S., Yang, Y., \& Cecil, G. 2001, ApJ, 560, 689

Young, A. J., \& Wilson, A. S. 2004, ApJ, 601, 133

Yuan, F., Markoff, S., Falcke, H., \& Biermann, P. L. 2002, A\&A, 391, 139 
Table 1. XMM-Newton EPIC OBSERVATIONS

\begin{tabular}{llccc}
\hline \hline $\begin{array}{c}\text { Observation } \\
\text { Date }\end{array}$ & Instrument & $\begin{array}{c}\text { Exposure } \\
\text { Time } \\
{[\mathrm{s}]}\end{array}$ & $\begin{array}{c}\text { Filtered } \\
\text { Exp. Time } \\
{[\mathrm{s}]}\end{array}$ & $\begin{array}{c}\text { Average } \\
\text { Count rate } \\
{\left[\mathrm{c} \mathrm{s}^{-1}\right]}\end{array}$ \\
\hline 2000-12-08 & & 14442 & 14442 & $0.734 \pm 0.007$ \\
& PN & 20250 & 20250 & $0.224 \pm 0.004$ \\
& MOS1 & 20261 & 20261 & $0.228 \pm 0.003$ \\
MOS2 & & & & \\
& PN & 8597 & 8597 & $0.623 \pm 0.009$ \\
& MOS1 & 11998 & 11998 & $0.180 \pm 0.004$ \\
& MOS2 & 12001 & 11985 & $0.182 \pm 0.004$ \\
& & & & \\
& PN & 9055 & 9055 & $0.568 \pm 0.008$ \\
& MOS1 & 12438 & 12438 & $0.166 \pm 0.004$ \\
& MOS2 & 12441 & 12441 & $0.166 \pm 0.004$ \\
& & & & \\
& PN $001-12-17$ & 7917 & 6850 & $0.523 \pm 0.009$ \\
& MOS1 & 12459 & 10783 & $0.147 \pm 0.004$ \\
& MOS2 & 12498 & 10584 & $0.147 \pm 0.004$ \\
& & & & \\
& PN & 12089 & 11755 & $0.420 \pm 0.007$ \\
& MOS1 & 15664 & 15461 & $0.113 \pm 0.003$ \\
& MOS2 & 15664 & 15664 & $0.122 \pm 0.003$ \\
\hline
\end{tabular}

aTotal "good" exposure time after filtering out times when the ratio of source counts to background count was less than 5 .

${ }^{\text {b}}$ This observation was also analyzed and published by Pietsch \& Read (2002). 
Table 2. SPECTRAL DATA FOR NGC $4258^{(a)}$

\begin{tabular}{|c|c|c|c|c|c|c|c|c|c|c|c|c|c|c|}
\hline Observatory & Date & $\begin{array}{c}k T \\
{[\mathrm{keV}]}\end{array}$ & $\begin{array}{l}f_{0.5-2}{ }^{(b)} \\
{\left[10^{-12}\right]}\end{array}$ & $\begin{array}{l}L_{0.5-2}(c) \\
{\left[10^{38}\right]}\end{array}$ & $\begin{array}{c}N_{H}(d) \\
{\left[10^{22} \mathrm{~cm}^{-2}\right]}\end{array}$ & $\Gamma_{p l}$ & $\begin{array}{c}f_{5-10}^{(b)} \\
{\left[10^{-12}\right]}\end{array}$ & $\begin{array}{l}L_{5-10}(c) \\
{\left[10^{40}\right]}\end{array}$ & $\begin{array}{c}f_{2-10}{ }^{(b)} \\
{\left[10^{-12}\right]}\end{array}$ & $\begin{array}{l}L_{2-10}{ }^{(c)} \\
{\left[10^{40}\right]}\end{array}$ & $\begin{array}{l}E_{\text {line }} \\
{[\mathrm{keV}]}\end{array}$ & $\begin{array}{c}E W_{\text {line }} \\
{[\mathrm{eV}]}\end{array}$ & $\begin{array}{c}f_{\text {line }}{ }^{(b)} \\
{\left[10^{-13}\right]}\end{array}$ & Ref. \\
\hline$A S C A$ & $\begin{array}{l}1993 \text { May } 05 \\
1993 \text { May } 05\end{array}$ & $0.5 \pm 0.2^{(e, f}$ & & & $\begin{array}{l}15 \pm 2 \\
13.6_{-2.2}^{+2.1}\end{array}$ & $\begin{array}{l}1.78 \pm 0.29 \\
1.78_{-0.26}^{+0.22}\end{array}$ & 5.1 & 4 & & 4.2 & $6.5 \pm 0.2$ & $250 \pm 100$ & & $\begin{array}{l}1 \\
2\end{array}$ \\
\hline $\begin{array}{l}A S C A \\
A S C A \\
A S C A \\
\text { Beppo-SAX }\end{array}$ & $\begin{array}{l}1996 \text { May } 23 \\
1996 \text { Jun } 5 \\
1996 \text { Dec } 18 \\
1998 \text { Dec } 19\end{array}$ & $0.6 \pm 0.1$ & $1.6^{(e)}$ & & $\begin{array}{l}9.2 \pm 0.9 \\
8.8 \pm_{-0.6}^{+0.7} \\
9.7 \pm 0.8 \\
9.4 \pm 1.2\end{array}$ & $\begin{array}{l}1.71_{-0.17}^{+0.18} \\
1.83 \pm 0.13 \\
1.87 \pm 0.15 \\
2.11 \pm 0.14\end{array}$ & $\begin{array}{l}8.3 \\
8.8 \\
9.5 \\
5.2\end{array}$ & $\begin{array}{l}6.1 \\
6.4 \\
6.9 \\
3.8\end{array}$ & 8 & 10 & $6.57 \pm 0.20$ & $85 \pm 65$ & & $\begin{array}{l}2 \\
2 \\
2 \\
3\end{array}$ \\
\hline$A S C A$ & $\begin{array}{l}1999 \text { May } 15 \\
1999 \text { May } 15\end{array}$ & $\begin{array}{l}0.47_{-0.09}^{+0.03} \\
0.36_{-0.02}^{+0.03}\end{array}$ & & & $\begin{array}{l}8.2 \pm 0.9 \\
9.5_{-0.9}^{+2.1}\end{array}$ & $\begin{array}{l}1.79_{-0.11}^{+0.31} \\
1.86_{-0.13}^{+0.40}\end{array}$ & 4.0 & 2.9 & & 5.8 & $6.45_{-0.07}^{+0.10}$ & $107_{-37}^{+42}$ & & $\begin{array}{l}2^{(g)} \\
2^{(h)}\end{array}$ \\
\hline Chandra & $\begin{array}{l}2000 \text { Mar } 08 \\
2000 \text { Mar } 08 \\
2000 \text { Mar } 08\end{array}$ & $\begin{array}{l}\mathrm{N} / \mathrm{A} \\
\mathrm{N} / \mathrm{A}\end{array}$ & $\begin{array}{l}\mathrm{N} / \mathrm{A} \\
\mathrm{N} / \mathrm{A}\end{array}$ & $\begin{array}{l}\mathrm{N} / \mathrm{A} \\
\mathrm{N} / \mathrm{A}\end{array}$ & $\begin{array}{l}7.2 \pm 1.8 \\
6.9_{-1.8}^{+2.2} \\
6.0_{-1.4}^{+1.8}\end{array}$ & $\begin{array}{l}1.4 \pm 0.5 \\
1.3 \pm 0.6 \\
1.0_{-0.4}^{+0.5}\end{array}$ & 15.9 & 11.0 & $\begin{array}{l}14.4 \\
21.2\end{array}$ & $\begin{array}{l}13.2 \\
12 \\
17.4\end{array}$ & $\begin{array}{l}6.4 \\
6.4\end{array}$ & $\begin{array}{l}<887 \\
<369\end{array}$ & $<12$ & $\begin{array}{l}4 \\
5 \\
6\end{array}$ \\
\hline Chandra & $\begin{array}{l}2000 \text { Apr } 17 \\
2000 \text { Apr } 17^{(i)}\end{array}$ & $\begin{array}{l}1.3_{-0.9}^{+\infty}(f) \\
0.5 \pm 0.1\end{array}$ & 0.07 & $\begin{array}{l}1.6 \\
1.7\end{array}$ & $\begin{array}{l}7.2_{-0.4}^{+0.7} \\
6.7_{-0.9}^{+1.0}\end{array}$ & $\begin{array}{l}1.5_{-0.0}^{+0.1} \\
1.4 \pm 0.3\end{array}$ & 8.3 & 5.8 & 11.8 & $\begin{array}{l}13 \\
10.6\end{array}$ & 6.4 & $<61$ & $<1.2$ & $\begin{array}{l}5 \\
6\end{array}$ \\
\hline XMM-Newton & $\begin{array}{l}2000 \text { Dec } 08 \\
2000 \text { Dec } 08\end{array}$ & $0.6 \pm 0.03$ & 0.2 & 14.6 & $\begin{array}{l}8.0 \pm 0.4 \\
8.6 \pm 0.4\end{array}$ & $\begin{array}{l}1.64 \pm 0.08 \\
1.7 \pm 0.1\end{array}$ & 6.1 & 4.4 & $\begin{array}{l}7.6 \\
9.0\end{array}$ & $\begin{array}{l}7.5 \\
9.1\end{array}$ & $\begin{array}{l}6.45 \\
6.4\end{array}$ & $\begin{array}{l}<40 \\
<69\end{array}$ & $<1.0$ & $\begin{array}{l}4 \\
6\end{array}$ \\
\hline XMM-Newton & 2001 May 06 & $0.5_{-0.05}^{+0.06}$ & 0.2 & 15.3 & $9.8 \pm 0.7$ & $1.9 \pm 0.1$ & 4.8 & 3.5 & 7.1 & 7.8 & 6.4 & $<49$ & $<0.6$ & 6 \\
\hline Chandra & $\begin{array}{l}2001 \text { May } 28 \\
2001 \text { May } 28^{(i)}\end{array}$ & $\begin{array}{l}1.3_{-0.8}^{+\infty}(f) \\
0.6_{-0.18}^{+\infty}\end{array}$ & 0.06 & $\begin{array}{l}1.4 \\
1.3\end{array}$ & $\begin{array}{l}6.5_{-0.3}^{+0.6} \\
6.0 \pm 0.7\end{array}$ & $\begin{array}{l}1.5 \pm 0.1 \\
1.4_{-0.2}^{+0.3}\end{array}$ & 6.6 & 4.5 & 9.4 & $\begin{array}{l}7.9 \\
8.1\end{array}$ & $\begin{array}{l}6.4 \\
6.4\end{array}$ & $\begin{array}{l}<132 \\
<191\end{array}$ & $<2.8$ & $\begin{array}{l}5 \\
6\end{array}$ \\
\hline Chandra & $\begin{array}{l}2001 \text { May } 29 \\
2001 \text { May } 29\end{array}$ & $\begin{array}{l}1.0_{-0.7}^{+0.6(f)} \\
0.8 \pm 0.2\end{array}$ & 0.06 & $\begin{array}{l}1.5 \\
1.5\end{array}$ & $\begin{array}{l}6.8_{-1.0}^{+1.2} \\
5.9_{-0.7}^{+0.8}\end{array}$ & $\begin{array}{l}1.4 \pm 0.3 \\
1.3 \pm 0.2\end{array}$ & 6.2 & 4.3 & 8.8 & $\begin{array}{l}7.4 \\
7.6\end{array}$ & $\begin{array}{l}6.4 \\
6.4\end{array}$ & $\begin{array}{l}<94 \\
<100\end{array}$ & $<1.4$ & $\begin{array}{l}5 \\
6\end{array}$ \\
\hline $\begin{array}{l}\text { XMM-Newton } \\
\text { XMM-Newton } \\
\text { XMM-Newton }\end{array}$ & $\begin{array}{l}2001 \text { Jun } 17 \\
2001 \text { Dec } 17 \\
2002 \text { May } 22\end{array}$ & $\begin{array}{l}0.5^{ \pm} 0.05 \\
0.5_{-0.12}^{+0.06} \\
0.5 \pm 0.04\end{array}$ & $\begin{array}{l}0.2 \\
0.3 \\
0.2\end{array}$ & $\begin{array}{l}14.1 \\
16.4 \\
14.9\end{array}$ & $\begin{array}{l}8.5_{-0.6}^{+0.7} \\
8.4_{-0.9}^{+1.0} \\
13.2_{-1.3}^{+1.4}\end{array}$ & $\begin{array}{l}1.8 \pm 0.1 \\
1.5_{-0.1}^{+0.2} \\
1.5 \pm 0.2\end{array}$ & $\begin{array}{l}4.3 \\
4.1 \\
3.7\end{array}$ & $\begin{array}{l}3.1 \\
2.9 \\
2.8\end{array}$ & $\begin{array}{l}6.4 \\
5.8 \\
4.9\end{array}$ & $\begin{array}{l}6.5 \\
5.5 \\
5.4\end{array}$ & $\begin{array}{l}6.3_{-0.08}^{+0.07(j)} \\
6.4 \\
6.4\end{array}$ & $\begin{array}{l}66 \pm 43^{(j)} \\
<67 \\
<100\end{array}$ & $\begin{array}{l}0.7 \\
<0.7 \\
<0.9\end{array}$ & $\begin{array}{l}6 \\
6 \\
6\end{array}$ \\
\hline
\end{tabular}

References- 1: Makishima et al. (1994), 2: Reynolds et al. (2000), 3: Fiore et al. (2001), 4: Pietsch and Read (2002), 5: Young and Wilson (2004), 6: this work

${ }^{(a)}$ Results reported here are from model fits performed on the 0.3-10 keV (XMM-Newton) and 0.5-10 keV(Chandra) energy ranges. The models comprise several components. For Chandra: Galactic absorption $\left(N_{H}(g a l)=1.19 \times 10^{20} \mathrm{~cm}^{-2}\right.$, frozen in the fits), intrinsic absorption $\left(N_{H}\right)$, low energy thermal plasma component $(k T)$, power law $\left(\Gamma_{p l}\right)$, and Gaussian Fe K-line $\left(E_{\text {line }}, W_{\text {line }}\right)$. For XMM-Newton an additional soft power law absorbed by $N_{H}(g a l)$ has also been added. The slope of this component remains almost constant

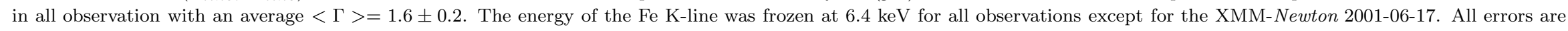
quoted at $90 \%$ confidence.

${ }^{(b)}$ Absorbed fluxes (ergs cm $\mathrm{s}^{-1}$ ) in the $0.5-2 \mathrm{keV}, 5-10 \mathrm{keV}$, and $2-10 \mathrm{keV}$ bands and Fe K-line flux or upper limit. For Beppo-SAX data, $f_{5-10}$ is derived via PIMMS assuming 
the published values of $f_{2-10}, N_{H}$, and $\Gamma_{p} l$

(c) Absorption-corrected luminosities (ergs s${ }^{-1}$ ) in the $0.5-2 \mathrm{keV}, 5-10 \mathrm{keV}$ and $2-10 \mathrm{keV}$ bands, assuming a distance of 7.2 Mpc. Both Galactic and intrinsic absorption have been removed. For $A S C A$ and Beppo-SAX data $L_{5-10}$ is derived via PIMMS assuming the values of $f_{5-10}, N_{H}$, and $\Gamma_{p} l$.

${ }^{(d)}$ Intrinsic equivalent hydrogen column density.

(e) $0.1-2.4 \mathrm{keV}$ energy band.

${ }^{(f)}$ Bremsstrahlung temperature.

(g) Model F (best fit) in Table 1 of Reynolds et al. (2000).

${ }^{(h)}$ Model G (with added Gaussian) in table 1 of Reynolds et al. (2000). Note that there are some typos in paragraph 3.2 of Reynolds et al. (2000): model D, model E, and model F should read model E, model F, and model G, respectively.

${ }^{(i)}$ A pile-up model with $\alpha$ and $f$ free to vary was added to the model components. See text and Table 3 for details.

${ }^{(j)}$ Values obtained by leaving $E_{\text {line }}$ free to vary. The fit is not significantly improved by the addition of a Gaussian line (reduced $\chi^{2}=1.05$ for 159 degrees of freedom without a line, reduced $\chi^{2}=1.02$ for 156 degrees of freedom with a line). 
Table 3. COMPARISON OF PILE-UP MODELING FOR CHANDRA OBSERVATIONS ${ }^{(a)}$

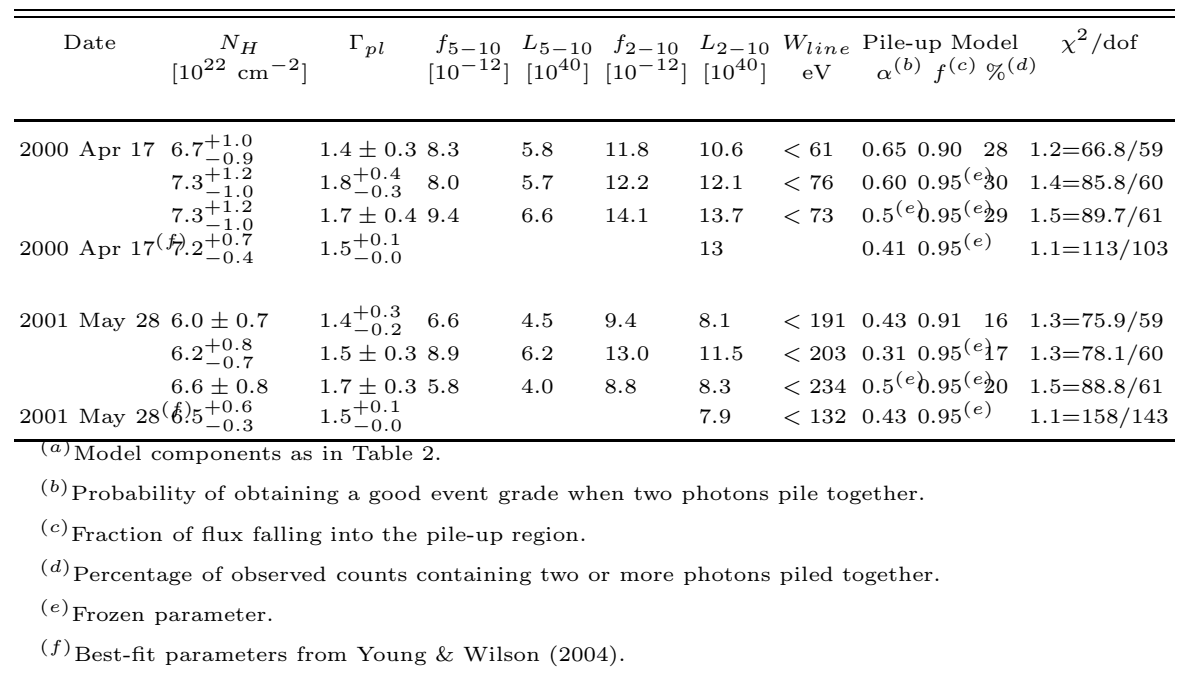




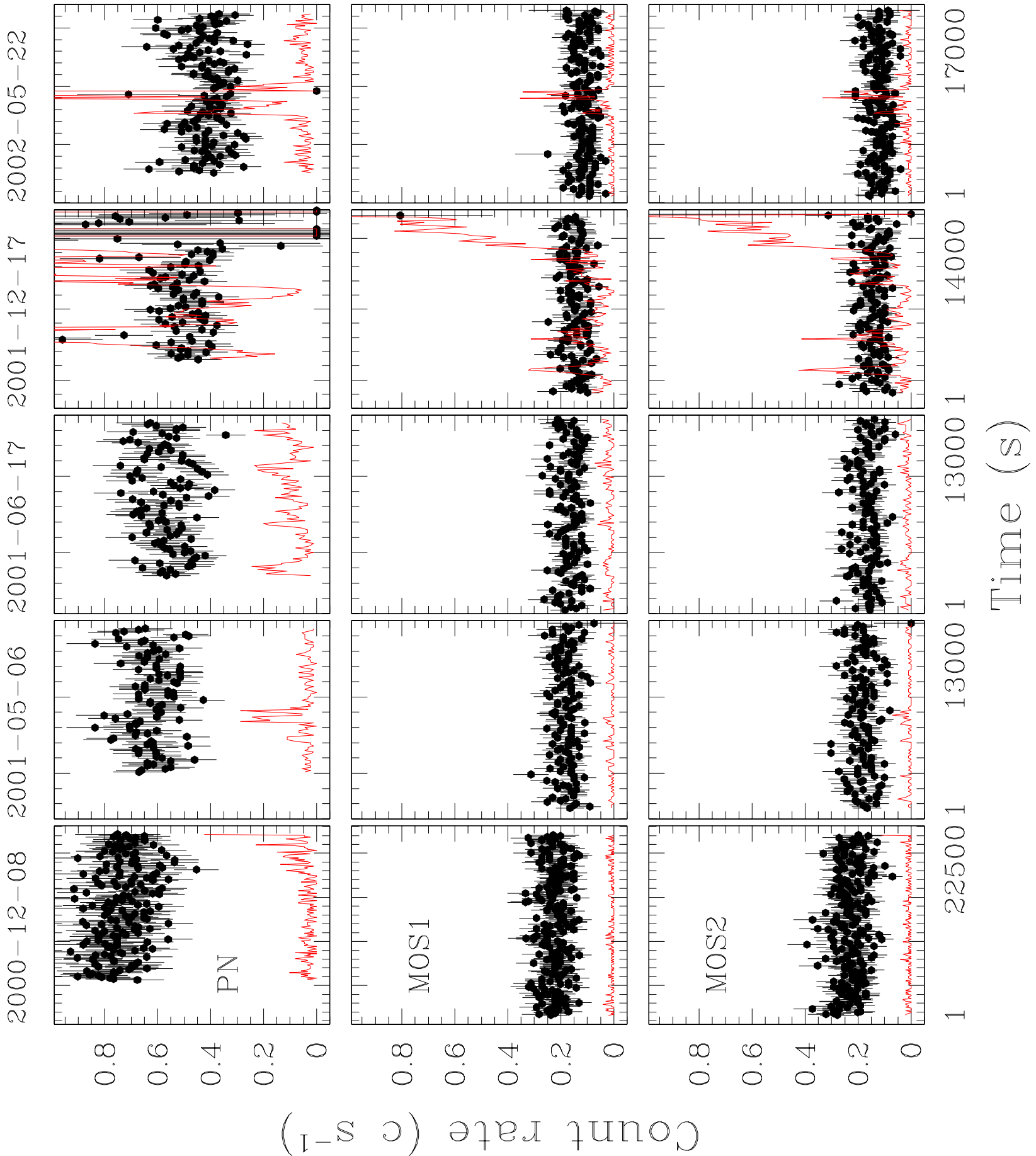

Fig. 1. - XMM-Newton EPIC background-subtracted light curves binned in $100 \mathrm{~s}$ bins for all five observation epochs. PN, MOS1, and MOS2 data are shown in the three rows. The continuous lines represent the level of the background. The light curves were extracted for the nuclear region of NGC 4258 ( $15^{\prime \prime}$ extraction radius) over the entire instrumental energy range (nominally $0.2-15 \mathrm{keV}$ ). Note that during epoch 4 (2001-12-17) the observation was affected by strong background flares and a generally very high background level. Also note that the time spacing is not uniform between the different epochs. 


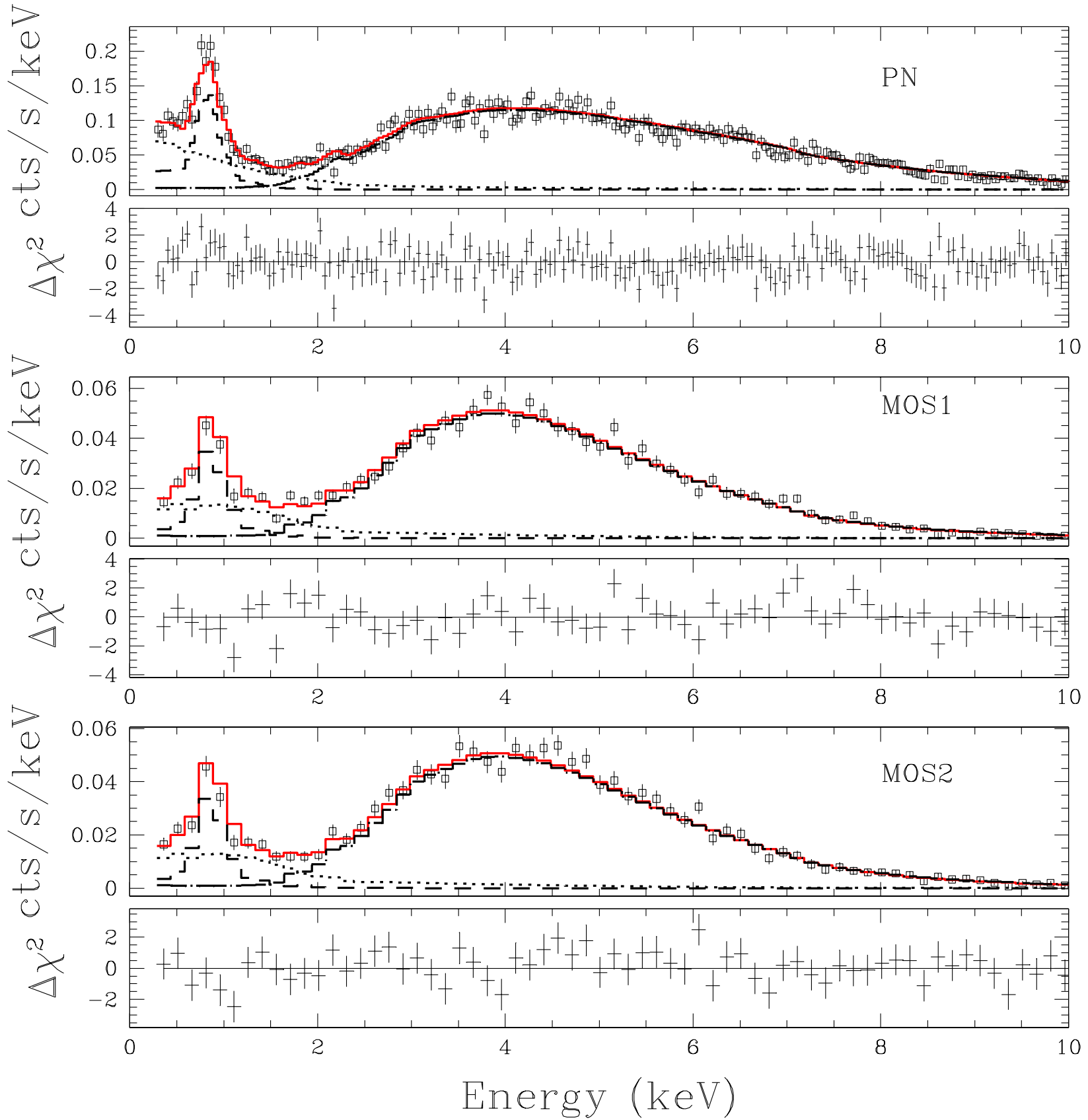

Fig. 2.- XMM-Newton EPIC spectra of the nucleus (15" extraction radius) of NGC 4258 for the observation of 2000-12-08. Data were "grouped" by 10 pulse-invariant (PI) channels. PN, MOS1, and MOS2 data were fitted simultaneously with a model consisting of 2 power laws (dotted and dot-dashed lines) plus a thermal spectrum (dashed line) absorbed by intrinsic and Galactic gas $\left(\mathrm{N}_{H}\right)$ (see text). The best fit (solid line) and residuals (in units of $\sigma$ ) are shown for all instruments. These are the same data presented by Pietsch \& Read (2002) (note that their Figures 2 and 5 report units of counts $\mathrm{cm}^{-2} \mathrm{~s}^{-1} \mathrm{keV}^{-1}$ while they should read counts $\mathrm{s}^{-1} \mathrm{keV}^{-1}$, as in our plots, given that the spectra are convolved with the instrumental ancillary response function). 


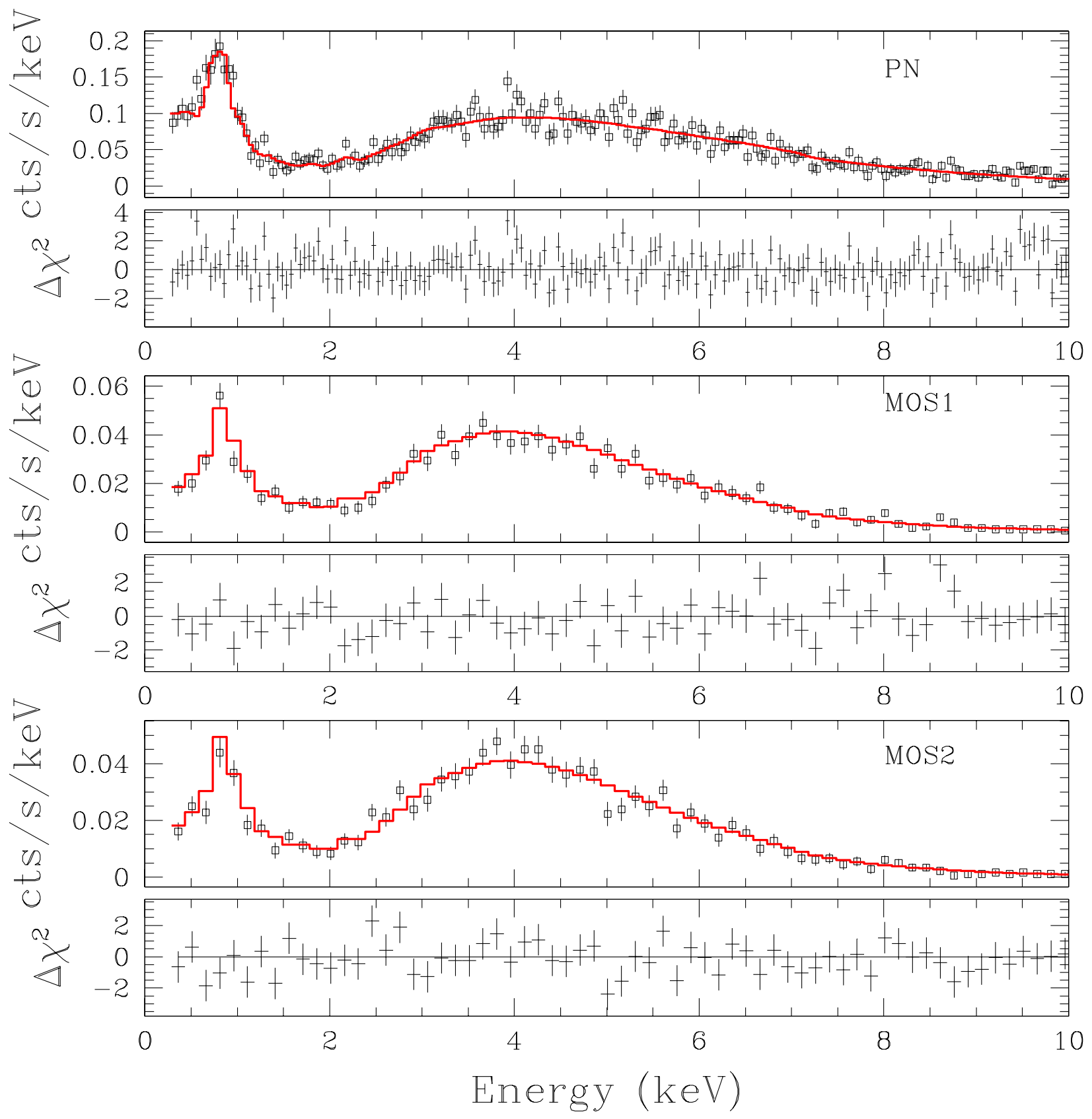

Fig. 3. - Same as Fig. 2 for the XMM-Newton observation of 2001-05-06. 


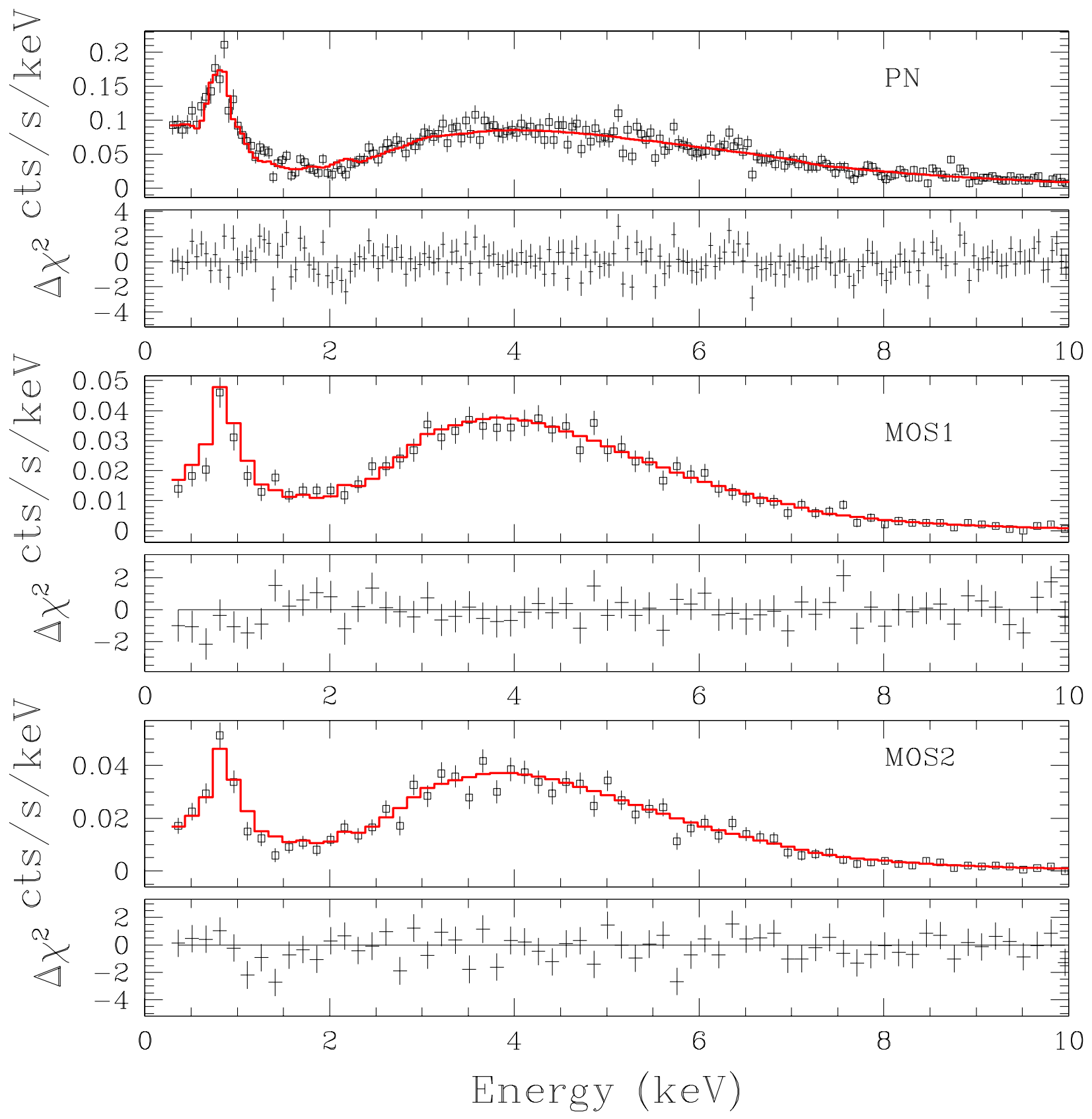

Fig. 4. - Same as Fig. 2 for the XMM-Newton observation of 2001-06-17. 


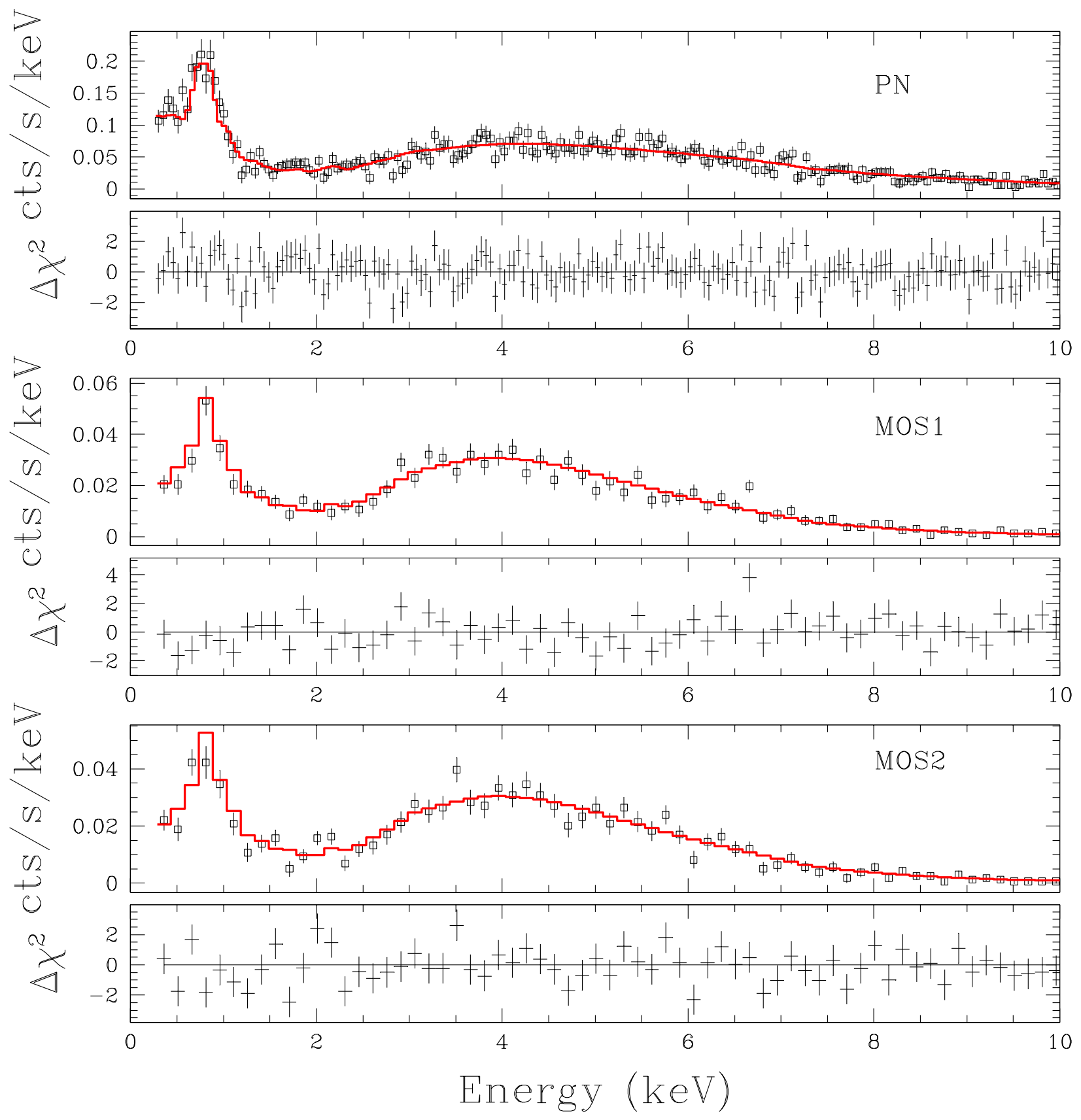

Fig. 5.- Same as Fig. 2 for the XMM-Newton observation of 2001-12-17. 


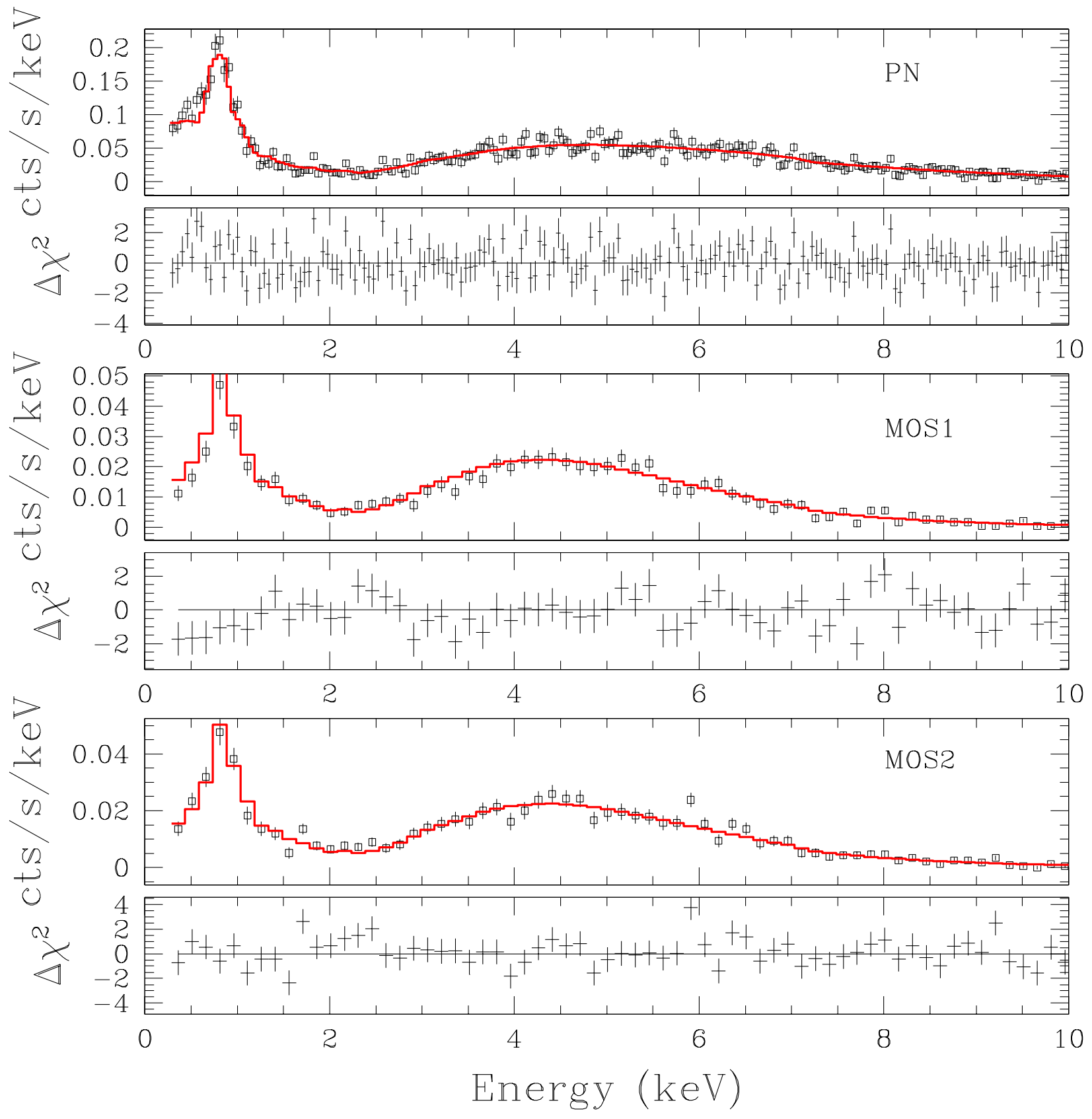

Fig. 6. - Same as Fig. 2 for the XMM-Newton observation of 2002-05-22. 

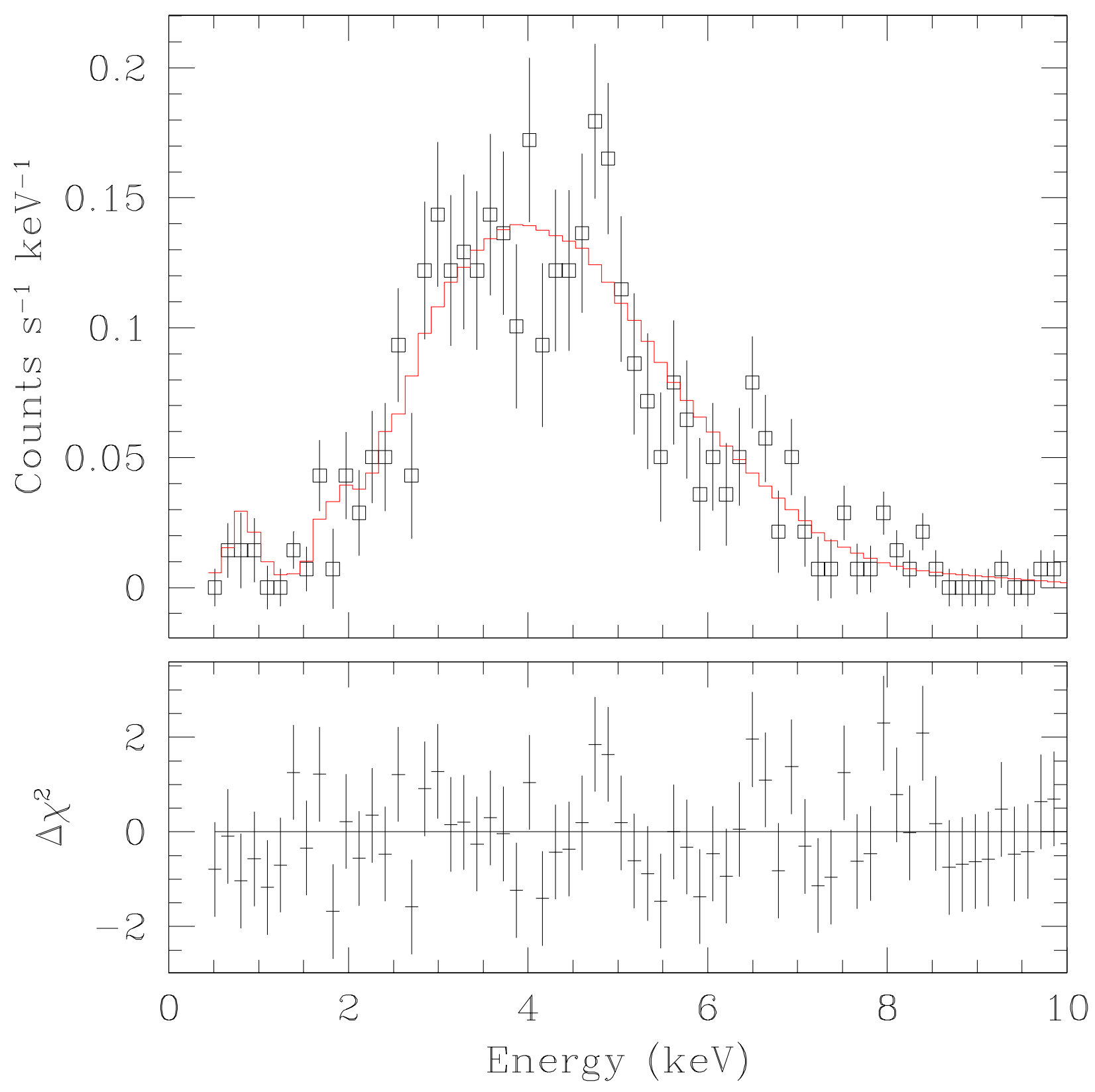

Fig. 7.- Chandra spectrum of the nucleus ( $2^{\prime \prime}$ extraction radius) of NGC 4258 for the observation of 2000-03-08. Data were "grouped" by 10 PI channels. The data were fitted with a model consisting of a power law plus a thermal component absorbed by intrinsic and Galactic gas $\left(\mathrm{N}_{H}\right)$ (see text). These are the same data presented by Pietsch \& Read (2002) (the final note of the caption in Fig. 2 applies). 

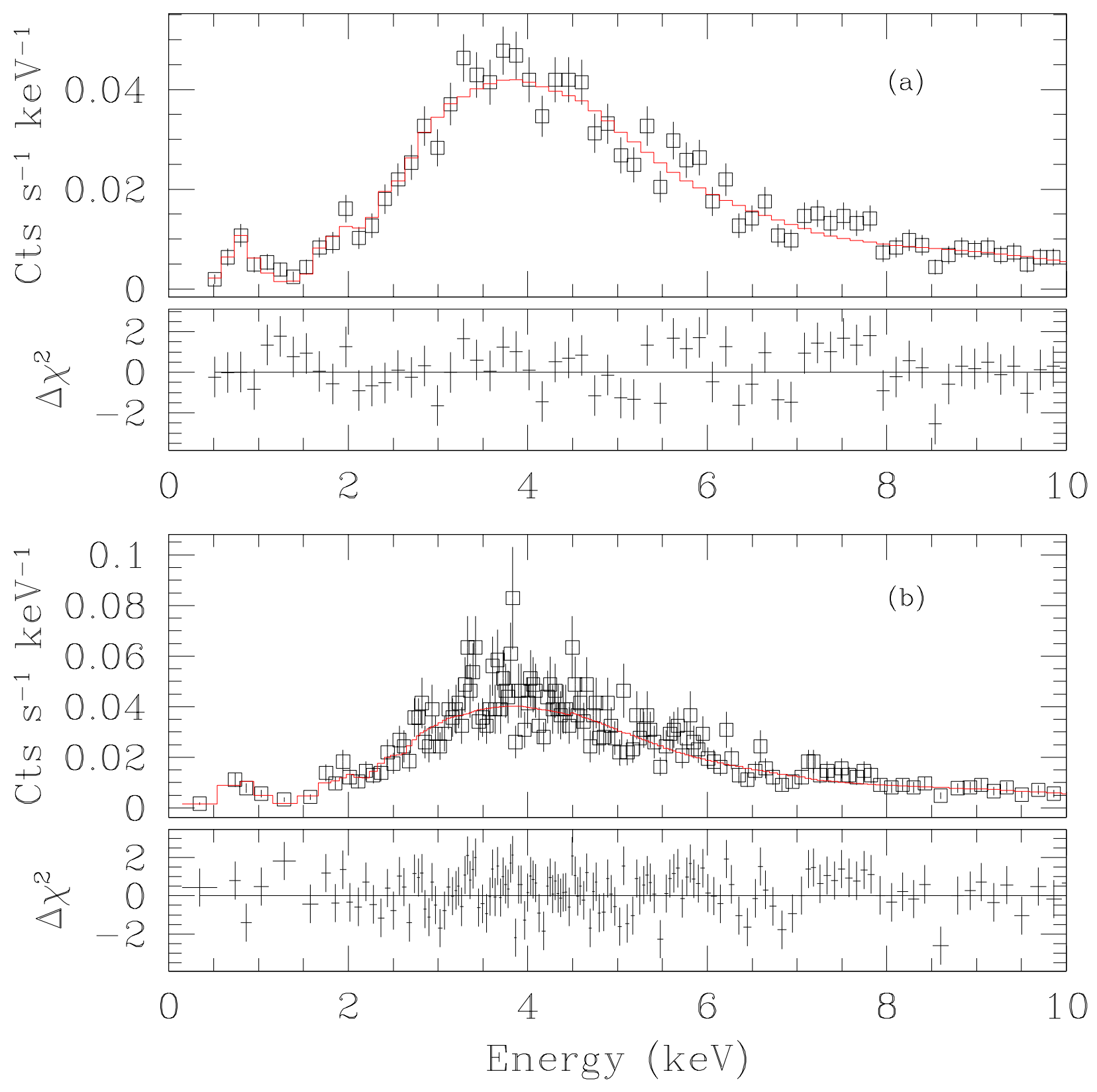

Fig. 8. - Same as Fig. 7 for the observation of 2000-04-17. This observation was affected by pile-up, of $\sim 30 \%$, which has been included in the modeling. Data were "grouped" by 10 pulse-invariant channels (panel $a$ ) or by a minimum number of counts of 15 per channel (panel $b$ ). The latter grouping scheme was the same as that used by Young \& Wilson (2004) for the same dataset. 

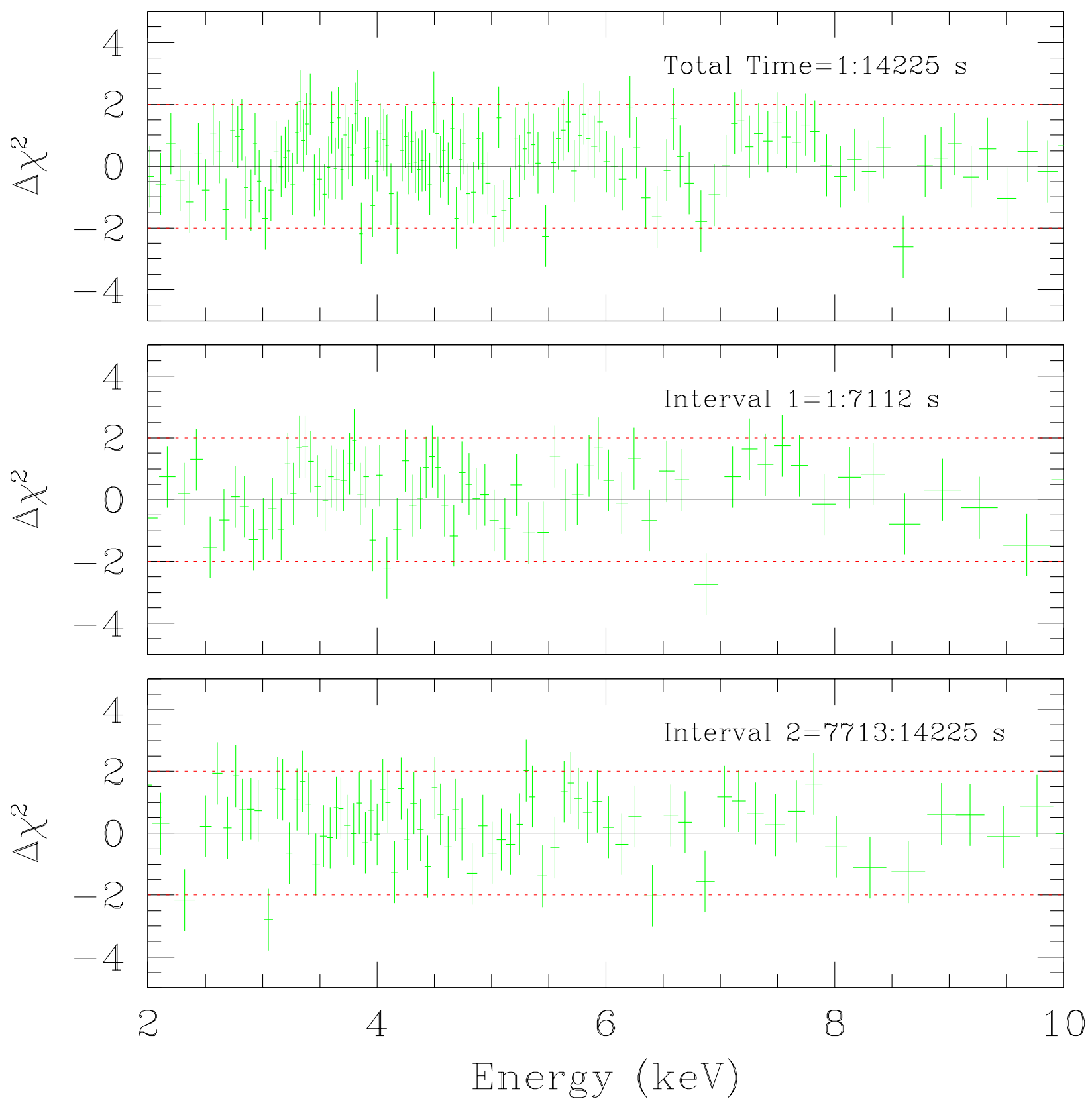

Fig. 9.- Residuals (in units of $\Delta \chi^{2}(\sigma)$ ) to an absorbed power law plus thermal plasma model for the 2000-04-17 observation. The figure compares directly to Figure 5 from Young \& Wilson (2004): data were grouped by a minimum number of counts per channel of 15 , and the three panels represent the entire set of observations, and the same divided into two equal $\sim 7000 \mathrm{~s}$ segments. The $2 \sigma$ levels are shown as dotted lines. 

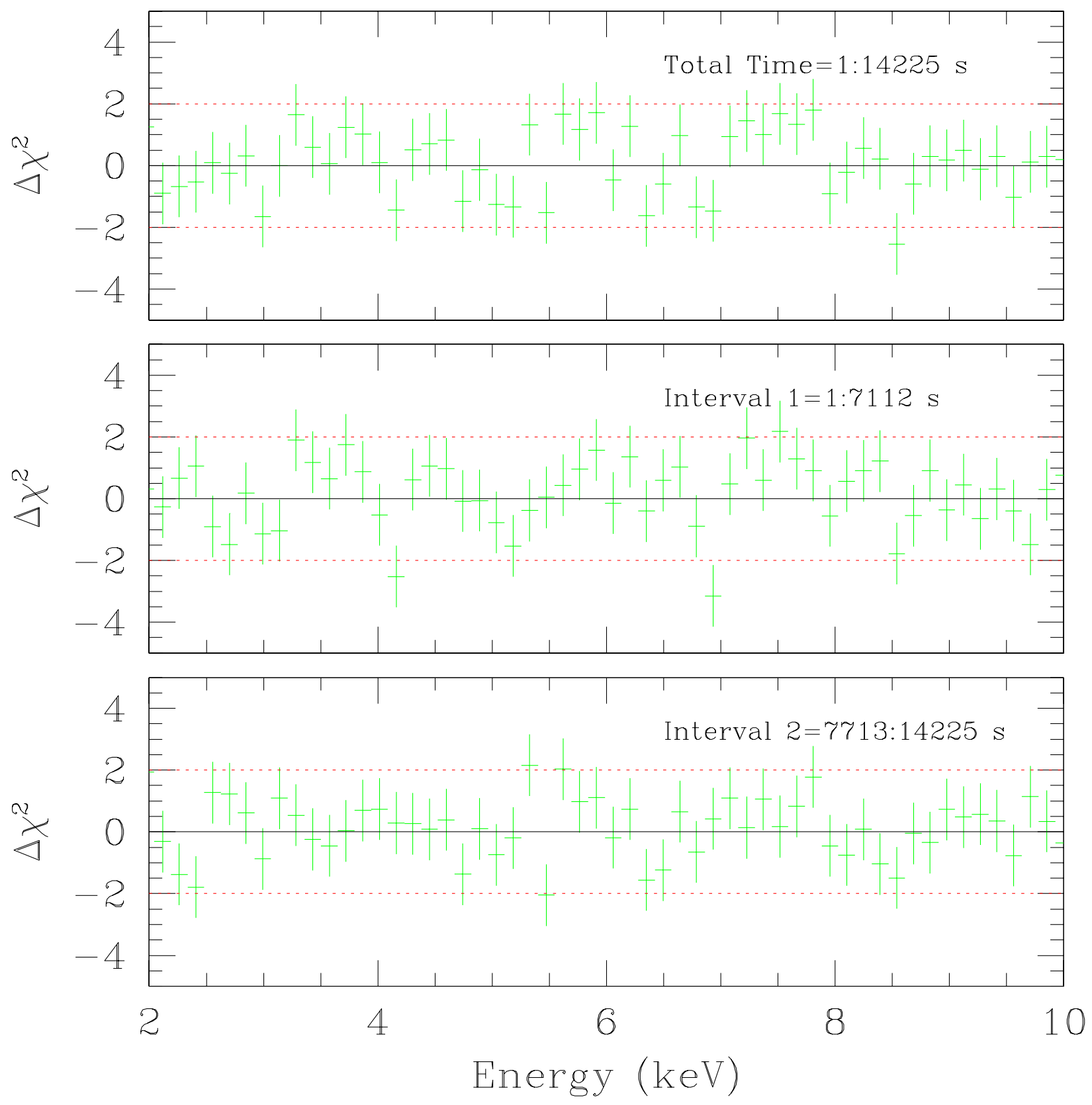

Fig. 10.- Same as Fig. 9 with grouping by 10 PI channels. 

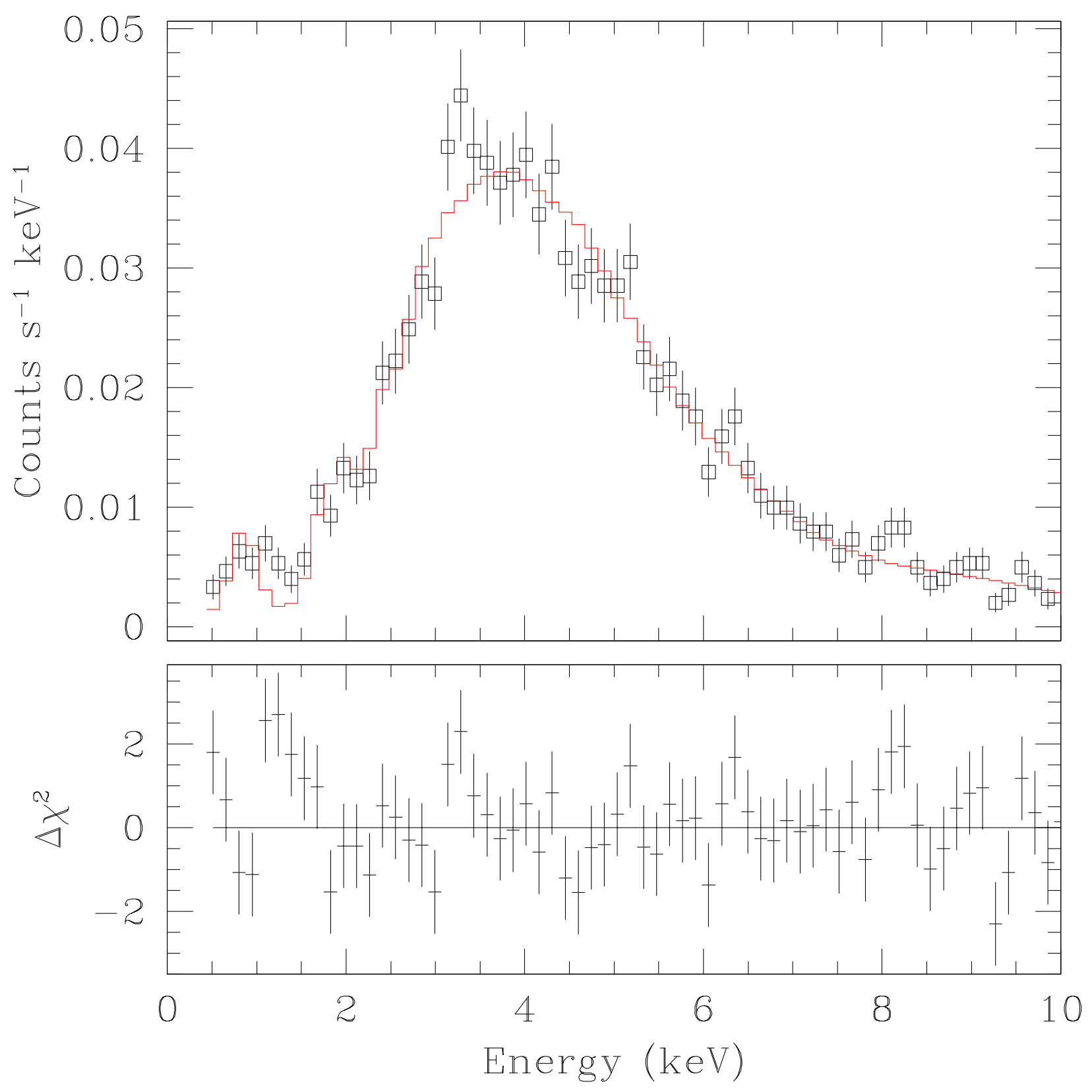

Fig. 11. - Same as Fig. 7 for the observation of 2001-05-28. This observation was affected by pile-up of $\sim 16 \%$. 

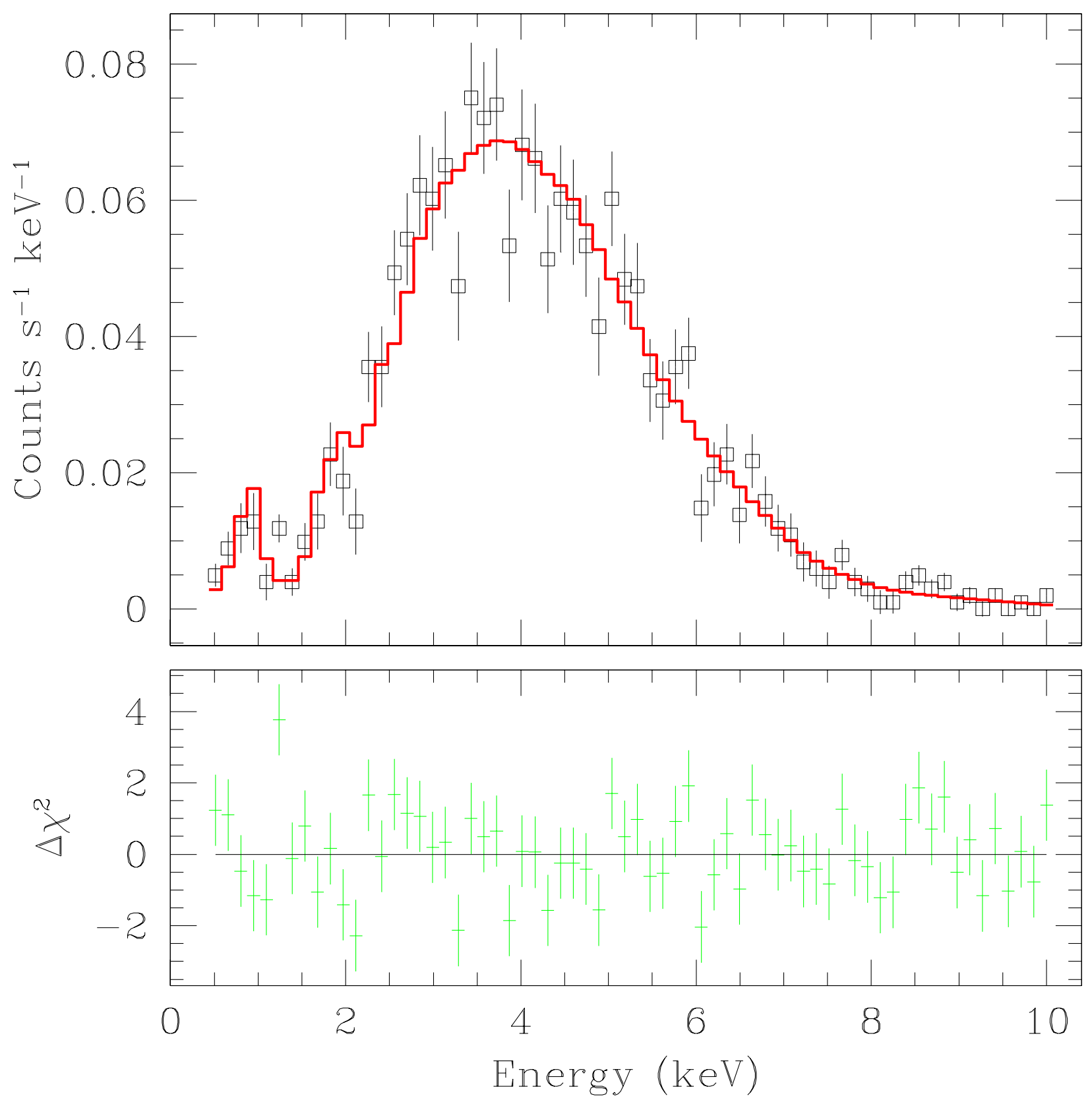

Fig. 12. - Same as Fig. 7 for the observation of 2001-05-29. 


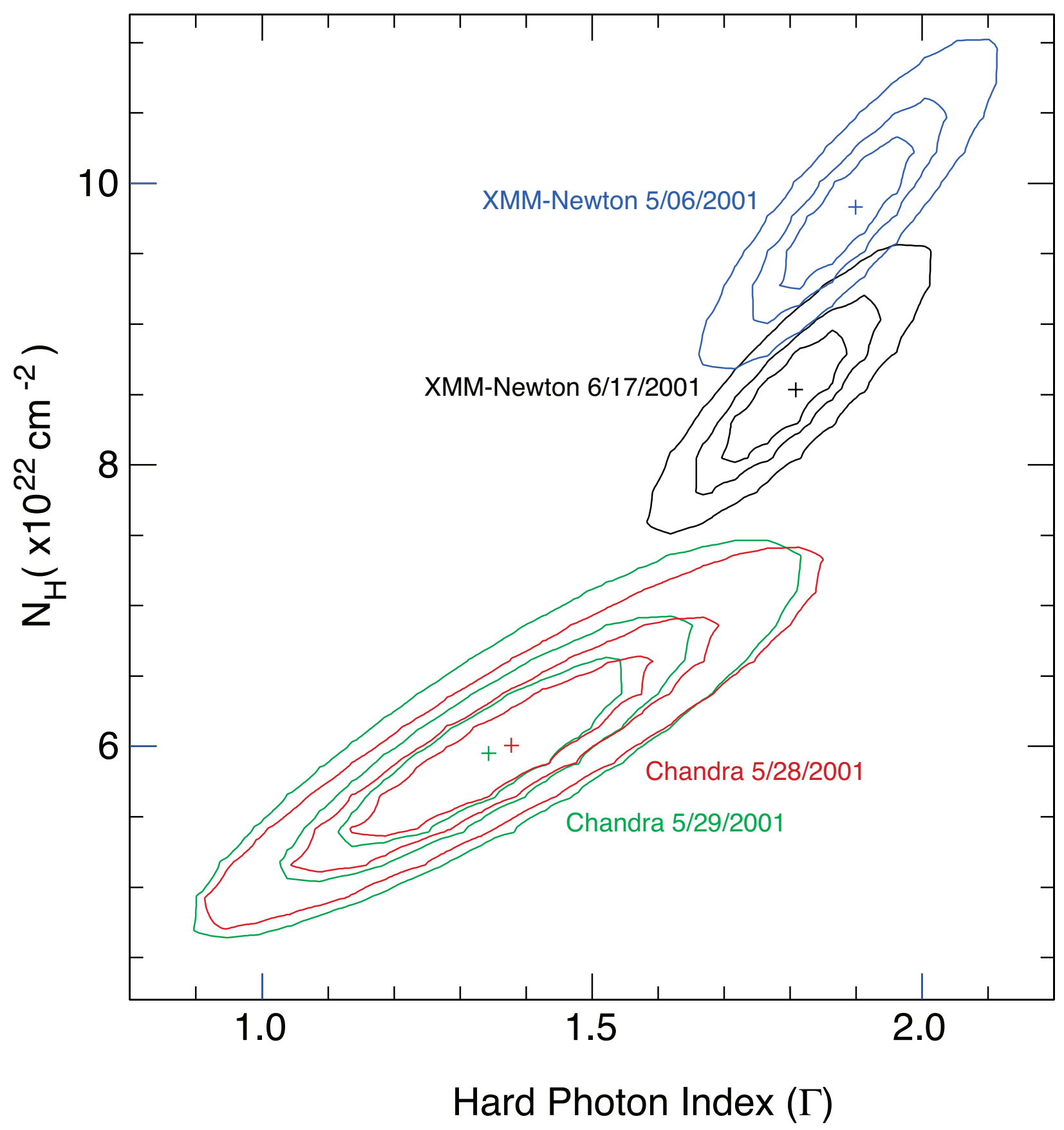

Fig. 13. - Contour plots of confidence regions for the model column density $\left(N_{H}\right)$ and photon index $(\Gamma)$ parameters. Contours for two sample XMM-Newton and Chandra observations are overlaid in the figure. Confidence intervals for each parameter are estimated independently and the three level represent confidence of $68.3 \%, 90 \%$ and $99 \%$ (corresponding to 1, 1.6 and $2.6 \sigma$ respectively). The plus sign marks the best-fit value. 

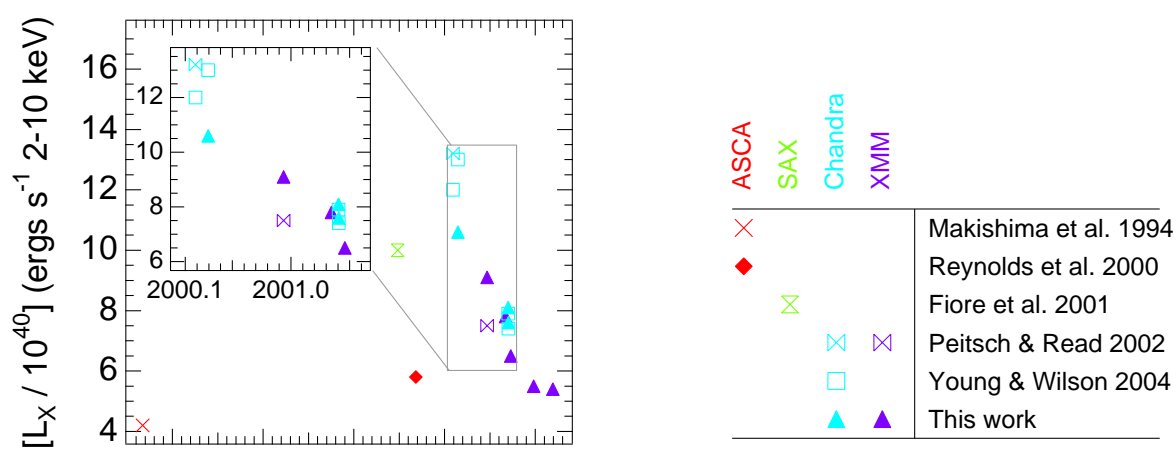

19941996199820002002

Epoch (year)
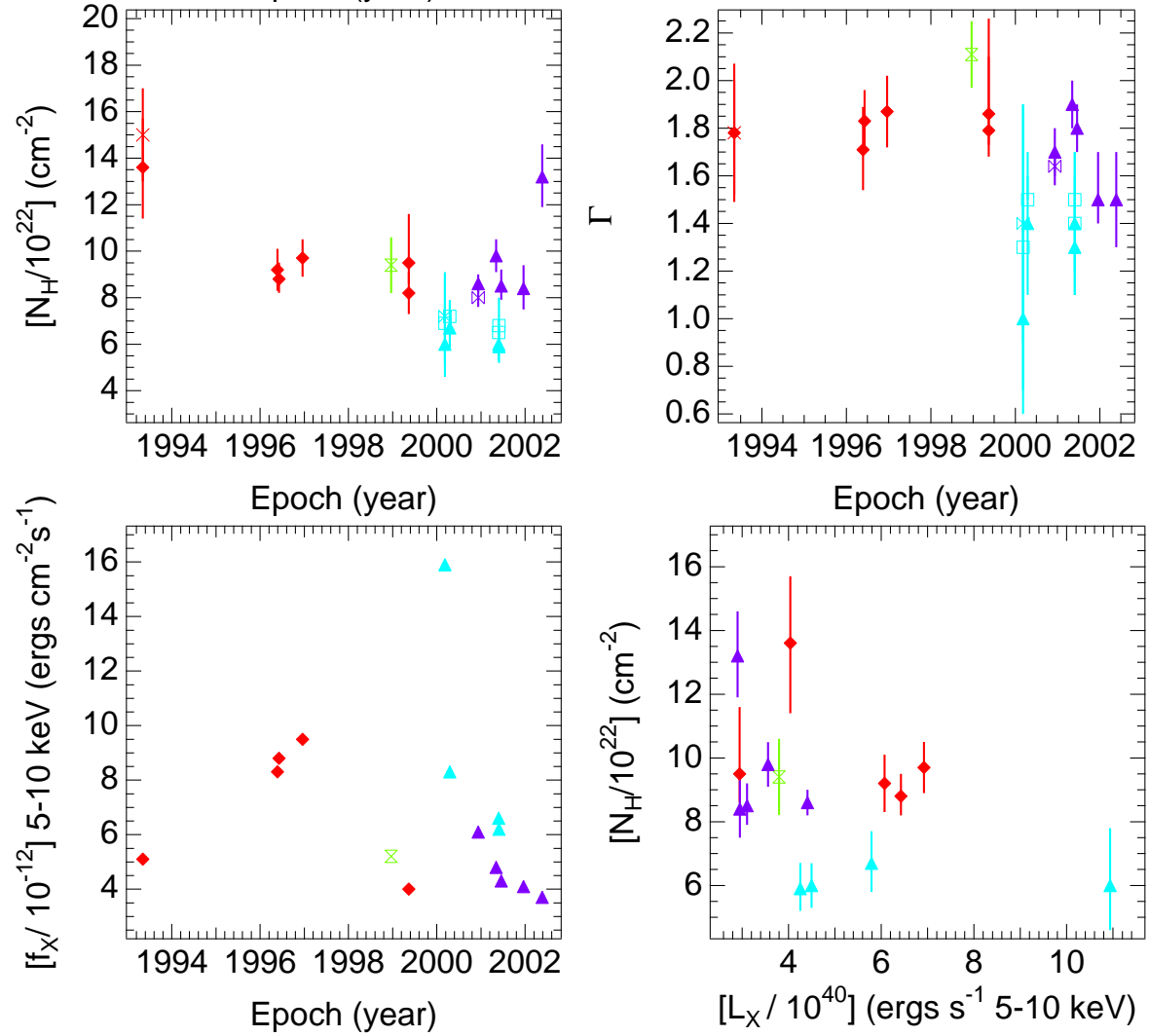

Fig. 14. - Time series of unabsorbed luminosity $\left(L_{X}\right)$, model column density $\left(N_{H}\right)$, photon index $(\Gamma)$, and absorbed flux $\left(f_{X}\right)$, as listed in Table 2. The lower right panel shows $N_{H}$ vs. hard $L_{X}$, which tracks the hard $f_{X}$ closely. No correlation is apparent, indicating that variations in flux are intrinsic to the central engine. Color is used to indicate mission while symbol type is used to code the reporting authors. Each dataset has been processed by more than one team. Plots of $L_{X}(2-10 \mathrm{keV})$ and $f_{X}(5-10 \mathrm{keV})$ are more sparse than plots of $N_{H}$ and $\Gamma$ because no single band is used throughout the literature. 